\title{
Theoretical UV Circular Dichroism of Aliphatic Cyclic Dipeptides
}

\section{Supplemental Information}

\section{Supplemental}

Figure 1

Figure 2

Figure 3

Figure 4

Figure 5

Figure 6

Figure 7

Figure 8

Figure 9

Figure 10

Figure 11

Figure 12

Figure 13

Figure 14

Figure 15

Figure 16

Figure 17

Figure 18

Figure 19

Figure 20

Figure 21

Figure 22

Figure 23

\section{Title}

Cyclo(L-Ala-L-Ala) CD Spectra, CVFF Geometries

Cyclo(L-Ala-L-Ala) CD Spectra, RHF Geometries

Cyclo(L-Ala-L-Ala) CD Spectra, B3LYP Geometries

Cyclo(L-Ala-L-Ala) CD Spectra, BLYP Geometries

Cyclo(L-Ala-L-Ala) CD Spectra, BVWN Geometries

Cyclo(L-Ala-L-Ala) CD Spectra, MP2 Geometries, AAll-I

Cyclo(L-Ala-L-Ala) CD Spectra, MP2 Geometries, AAll-II

Cyclo(L-Pro-Gly) CD Spectra, CVFF Geometries

Cyclo(L-Pro-Gly) CD Spectra, RHF Geometries

Cyclo(L-Pro-Gly) CD Spectra, B3LYP Geometries

Cyclo(L-Pro-Gly) CD Spectra, BLYP Geometries

Cyclo(L-Pro-Gly) CD Spectra, BVWN Geometries

Cyclo(L-Pro-Gly) CD Spectra, MP2 Geometries

Cyclo(L-Pro-L-Ala) CD Spectra, CVFF Geometries

Cyclo(L-Pro-L-Ala) CD Spectra, RHF Geometries

Cyclo(L-Pro-L-Ala) CD Spectra, B3LYP Geometries

Cyclo(L-Pro-L-Ala) CD Spectra, BLYP Geometries

Cyclo(L-Pro-L-Ala) CD Spectra, BVWN Geometries

Cyclo(L-Pro-L-Ala) CD Spectra, MP2 Geometries

Cyclo(L-Pro-L-Leu) CD Spectra, CVFF Geometries

Cyclo(L-Pro-L-Leu) CD Spectra, RHF Geometries

Cyclo(L-Pro-L-Leu) CD Spectra, B3LYP Geometries

Cyclo(L-Pro-L-Leu) CD Spectra, BLYP Geometries 
Figure 24 Cyclo(L-Pro-L-Leu) CD Spectra, BVWN Geometries

Figure $25 \quad$ Cyclo(L-Pro-L-Leu) CD Spectra, MP2 Geometries

Table $1 \quad$ Cyclo(L-Ala-L-Ala) Bond Lengths

Table 2 Cyclo(L-Ala-L-Ala) Bond Angles

Table 3 Cyclo(L-Ala-L-Ala) Bond Dihedral Angles

Table 4 Cyclo(L-Pro-Gly) Bond Lengths

Table $5 \quad$ Cyclo(L-Pro-Gly) Bond Angles

Table $6 \quad$ Cyclo(L-Pro-Gly) Bond Dihedral Angles

Table $7 \quad$ Cyclo(L-Pro-L-Ala) Bond Lengths

Table $8 \quad$ Cyclo(L-Pro-L-Ala) Bond Angles

Table 9 Cyclo(L-Pro-L-Ala) Bond Dihedral Angles

Table $10 \quad$ Cyclo(L-Pro-L-Leu) Bond Lengths

Table $11 \quad$ Cyclo(L-Pro-L-Leu) Bond Angles

Table 12 Cyclo(L-Pro-L-Leu) Bond Dihedral Angles 
Supplement Figure 1. Cyclo(L-Ala-L-Ala)I CD Spectra, CVFF Geometries

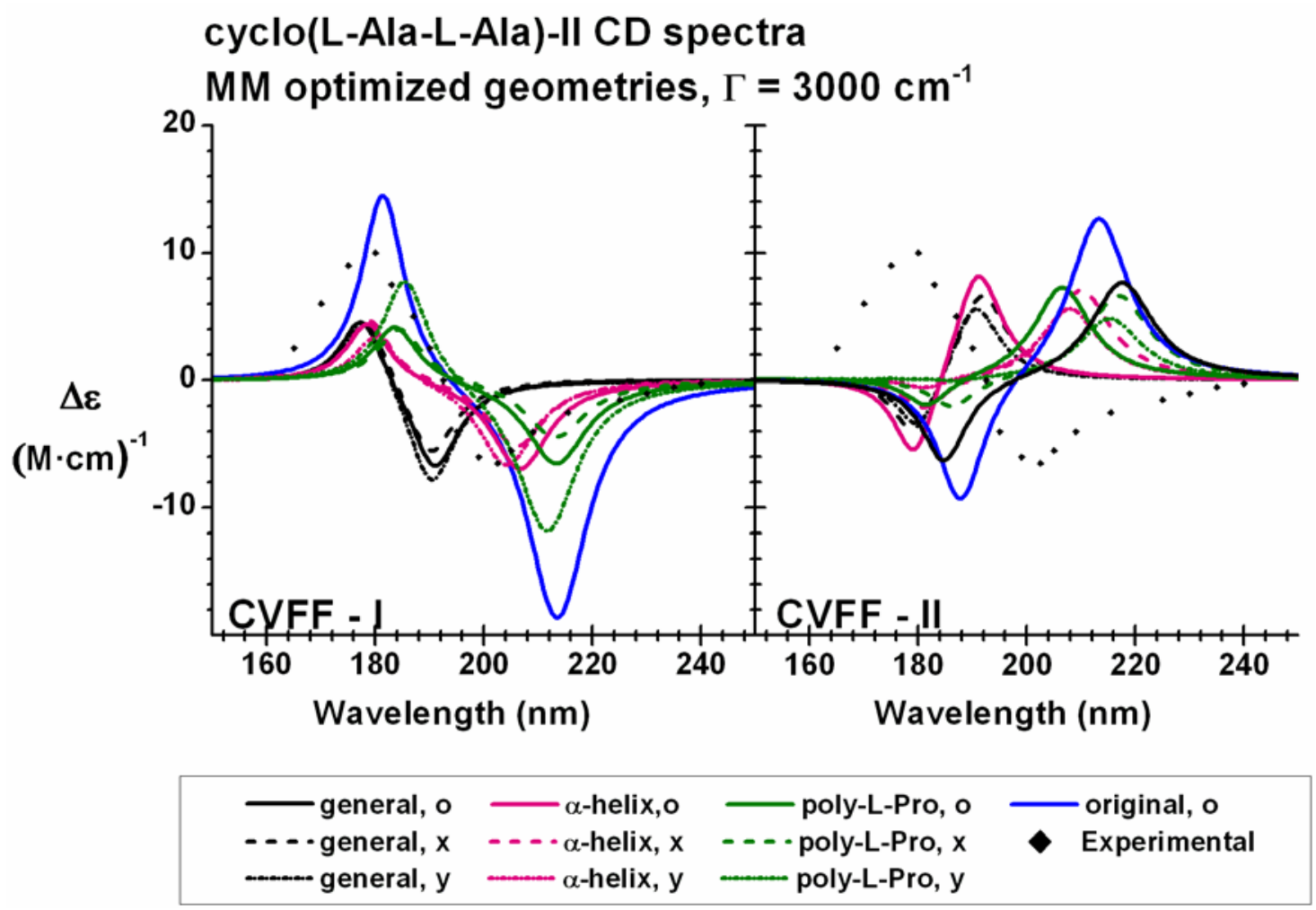

The experimental CD (in water) was obtained from [Bowman, R. L.; Kellerman, M.; Johnson, W. C., Jr. Biopolymers 1983, 22, 1045]. 
Supplement Figure 2. Cyclo(L-Ala-L-Ala)I CD Spectra, RHF Geometries

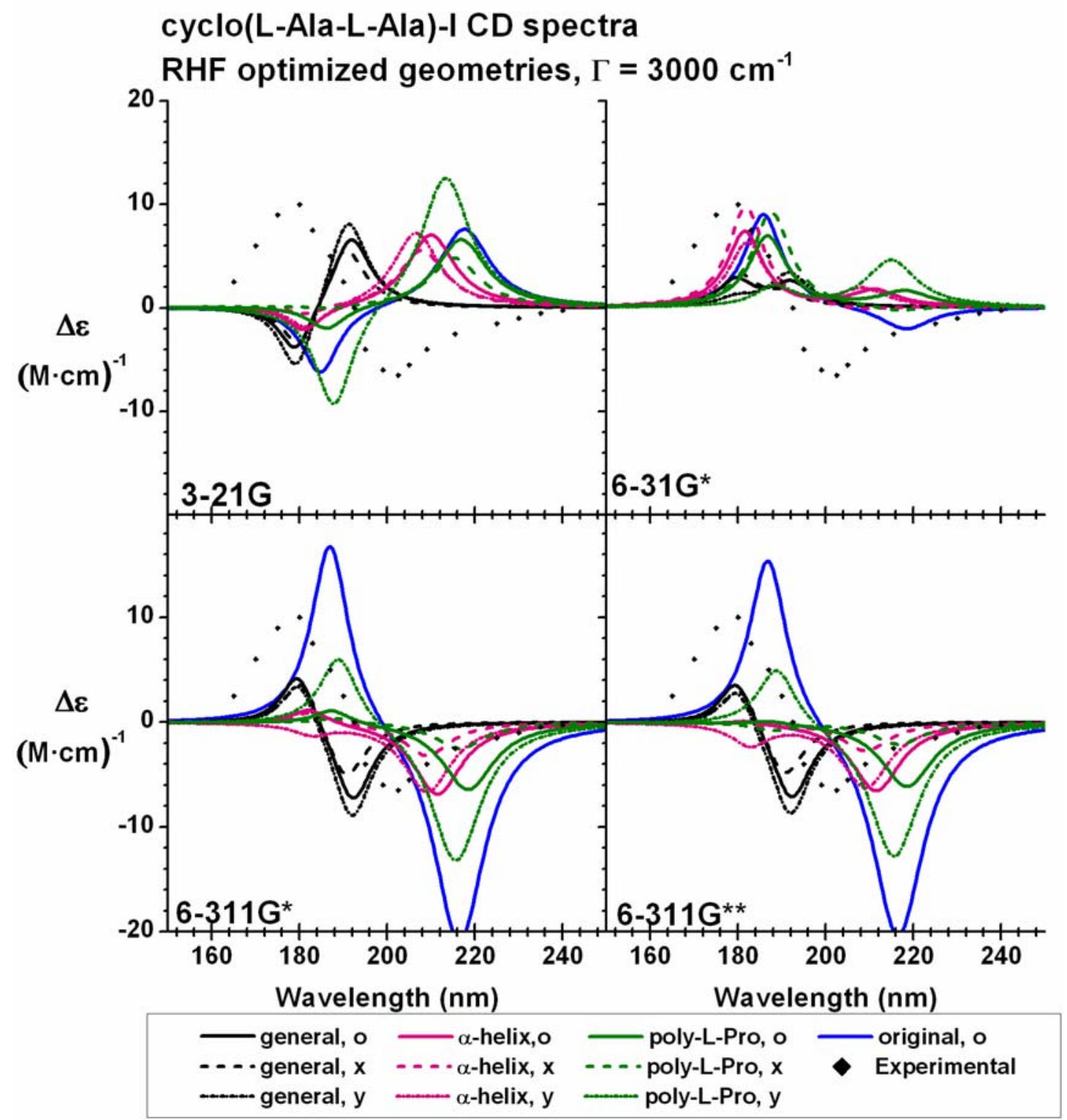

The experimental CD (in water) was obtained from [Bowman, R. L.; Kellerman, M.; Johnson, W. C., Jr. Biopolymers 1983, 22, 1045]. 
Supplement Figure 3. Cyclo(L-Ala-L-Ala)I CD Spectra, B3LYP Geometries

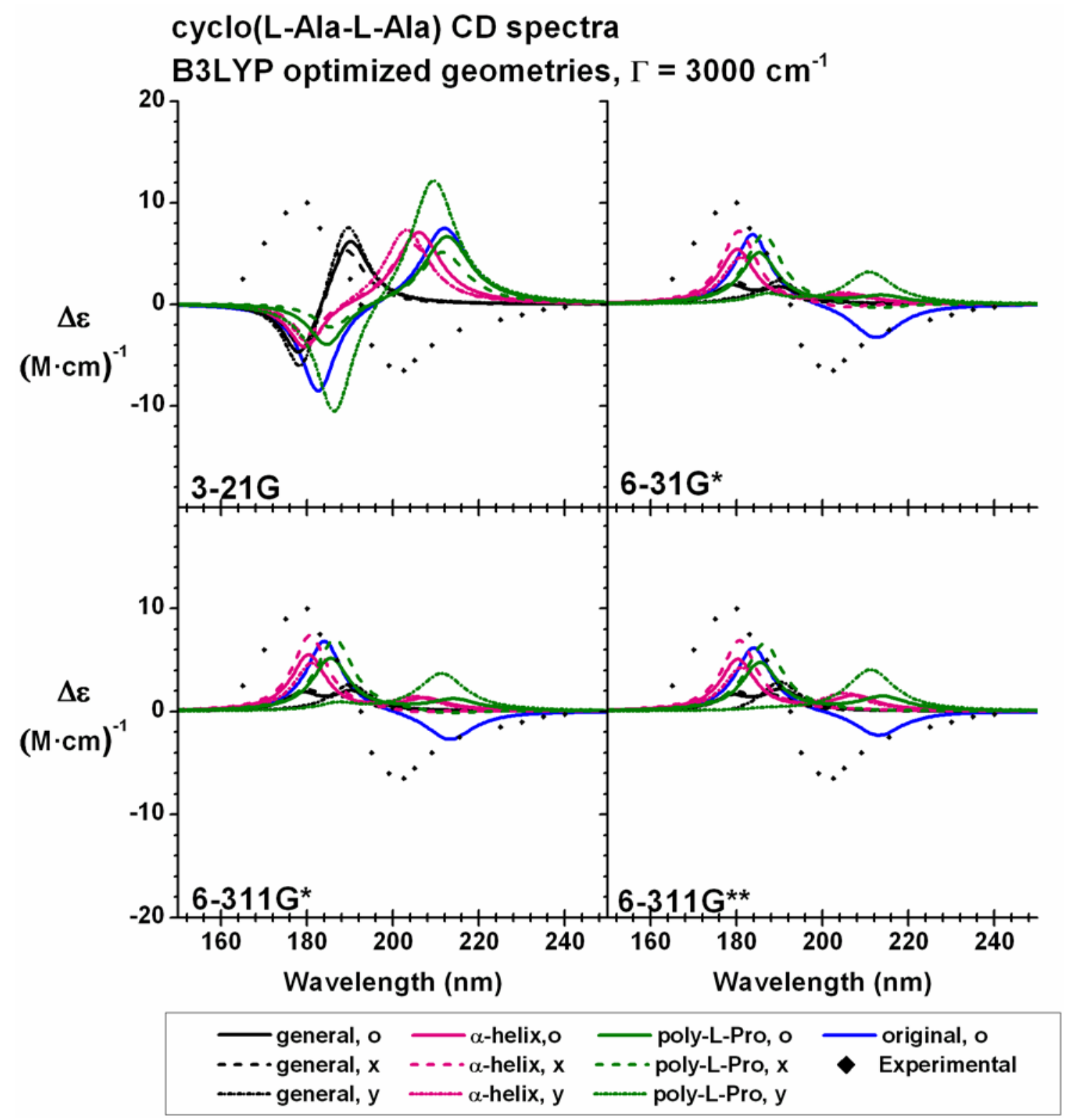

The experimental CD (in water) was obtained from [Bowman, R. L.; Kellerman, M.; Johnson, W. C., Jr. Biopolymers 1983, 22, 1045]. 
Supplement Figure 4. Cyclo(L-Ala-L-Ala)I CD Spectra, BLYP Geometries

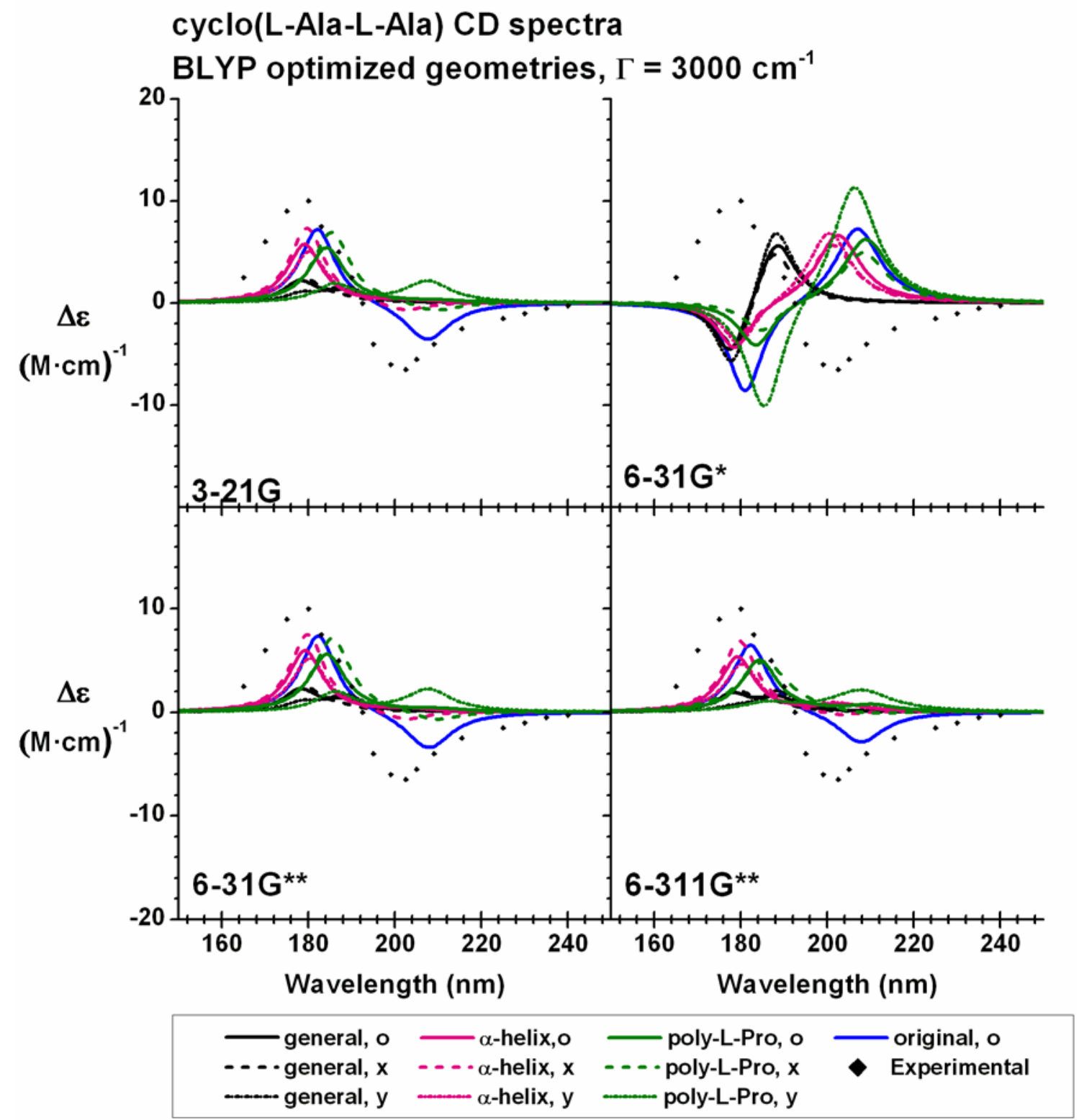

The experimental CD (in water) was obtained from [Bowman, R. L.; Kellerman, M.; Johnson, W. C., Jr. Biopolymers 1983, 22, 1045]. 
Supplement Figure 5. Cyclo(L-Ala-L-Ala)I CD Spectra, BVWN Geometries

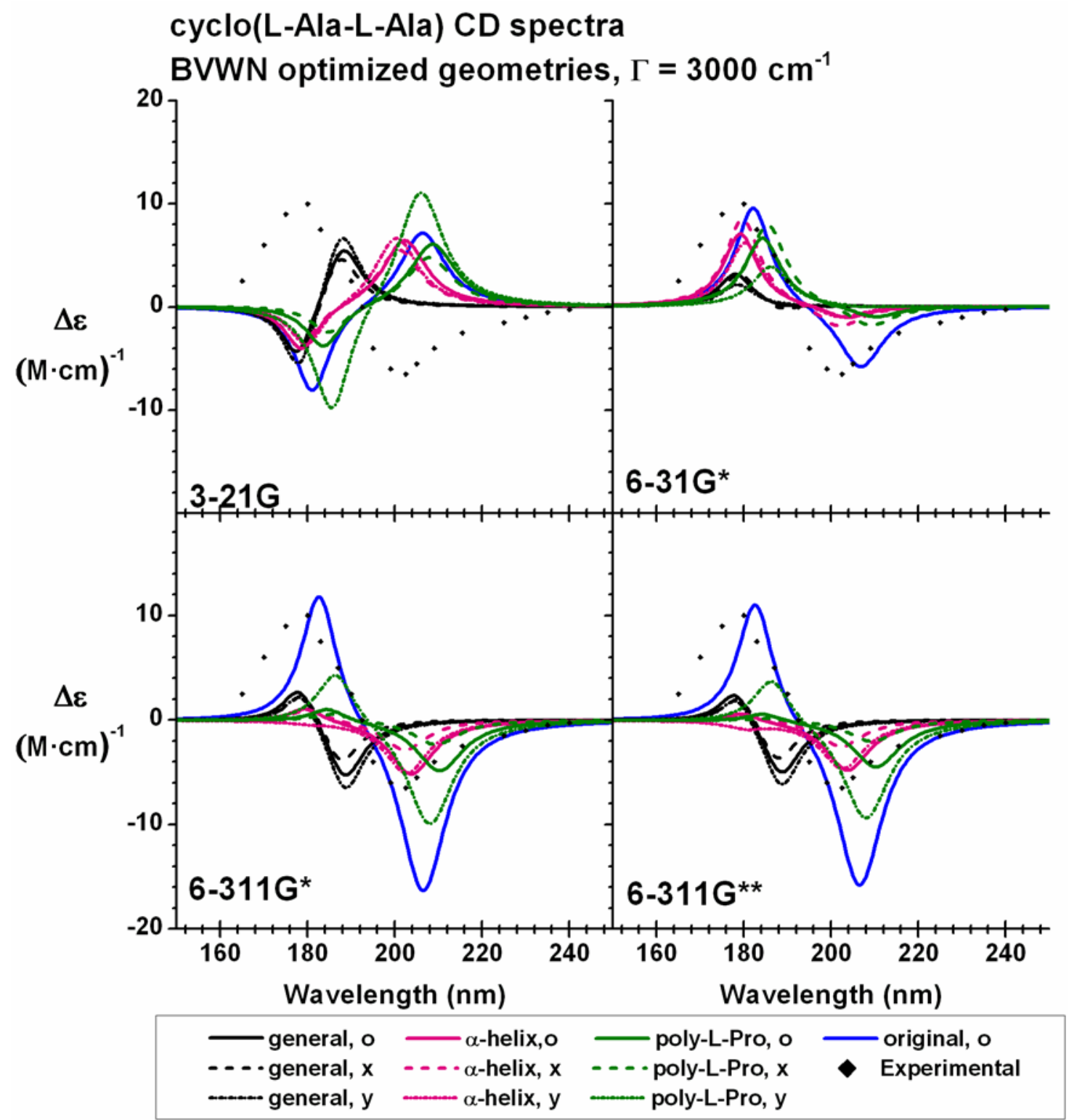

The experimental CD (in water) was obtained from [Bowman, R. L.; Kellerman, M.; Johnson, W. C., Jr. Biopolymers 1983, 22, 1045]. 
Supplement Figure 6. Cyclo(L-Ala-L-Ala)I CD Spectra, MP2 Geometries, AAll-I

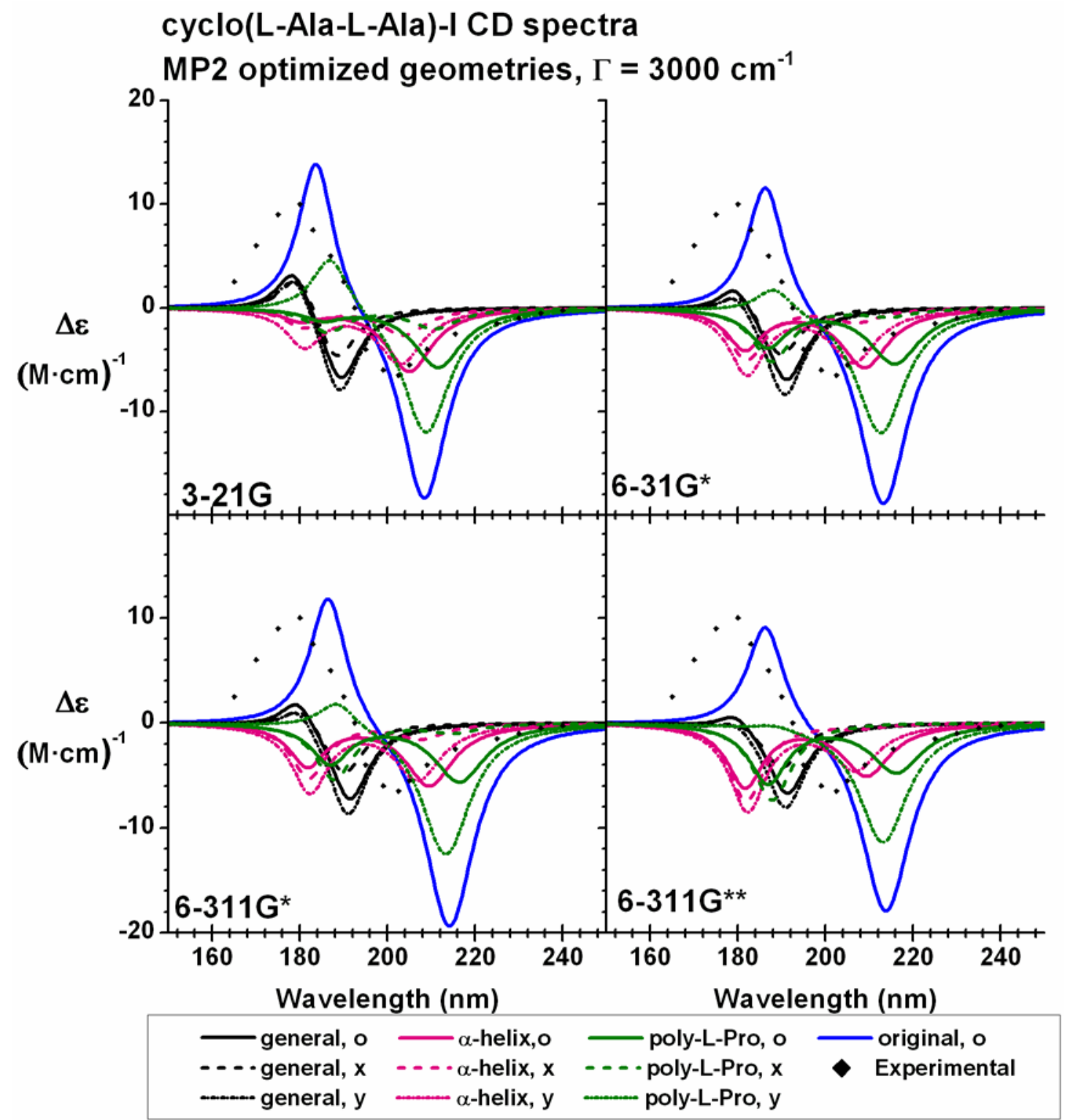

The experimental CD (in water) was obtained from [Bowman, R. L.; Kellerman, M.; Johnson, W. C., Jr. Biopolymers 1983, 22, 1045]. 
Supplement Figure 7. Cyclo(L-Ala-L-Ala)I CD Spectra, MP2 Geometries, AAll-II

\section{cyclo(L-Ala-L-Ala)-II CD spectra}

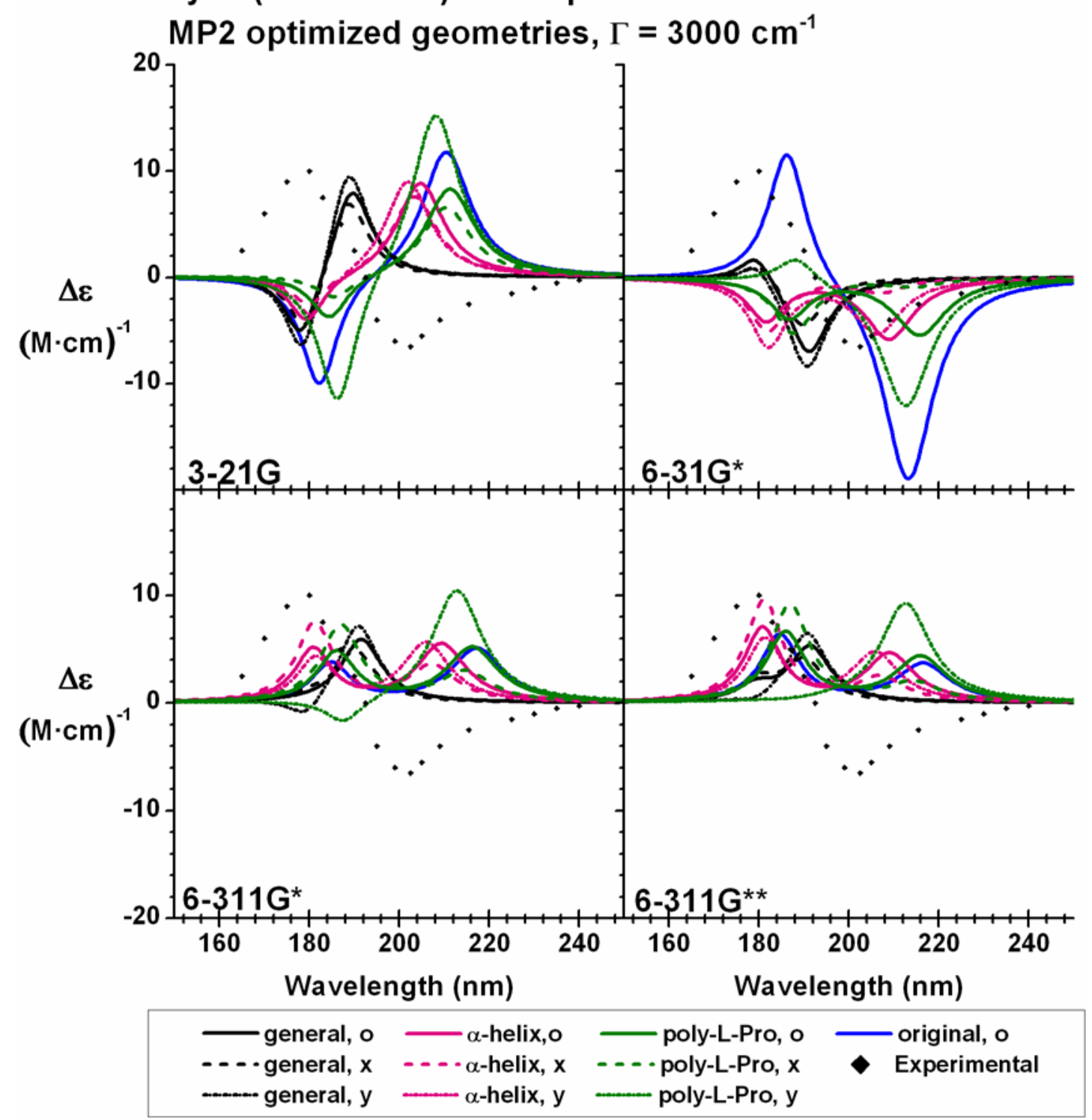

The experimental CD (in water) was obtained from [Bowman, R. L.; Kellerman, M.; Johnson, W. C., Jr. Biopolymers 1983, 22, 1045]. 
Supplement Figure 8. Cyclo(L-Pro-Gly) Calculated CD, CVFF Geometries

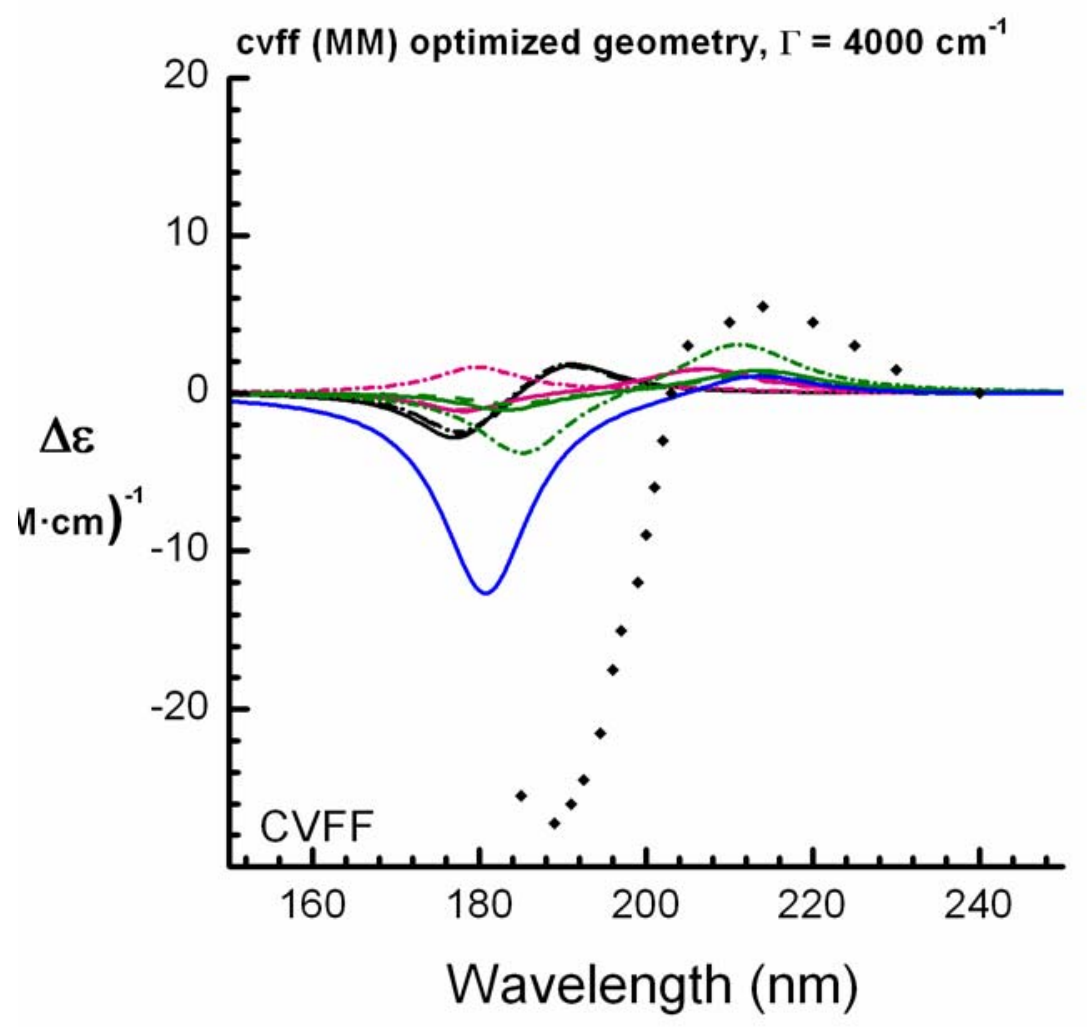

$$
\begin{aligned}
& \text { - general, o - }-\alpha \text {-helix,o poly-L-Pro, o - original, o } \\
& \text { - - general, } x \quad-\quad \alpha \text {-helix, } x \quad-\text { poly-L-Pro, } x \diamond \text { Experimental }
\end{aligned}
$$

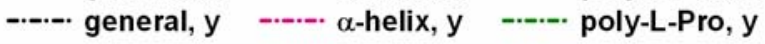

The experimental CD (in trifluoroethanol) was obtained from [Pancoska, P.; Fric, I.; Blaha, K. Coll. Czech.Chem. Comm. 1979, 44, 1296.] 
Supplement Figure 9. Cyclo(L-Pro-Gly) Calculated CD, RHF Geometries

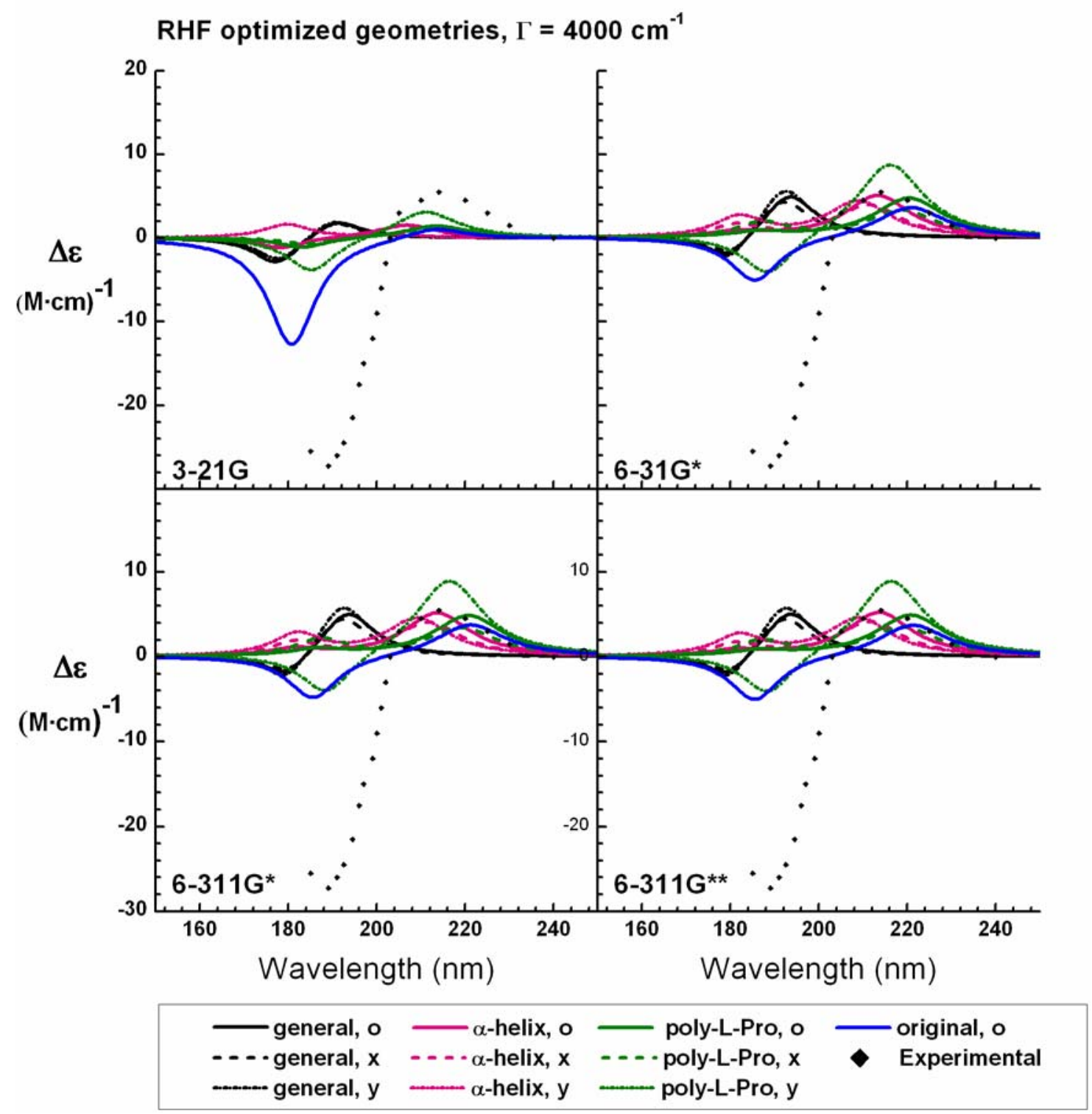

The experimental CD (in trifluoroethanol) was obtained from [Pancoska, P.; Fric, I.; Blaha, K. Coll. Czech.Chem. Comm. 1979, 44, 1296.] 
Supplement Figure 10. Cyclo(L-Pro-Gly) Calculated CD, B3LYP Geometries

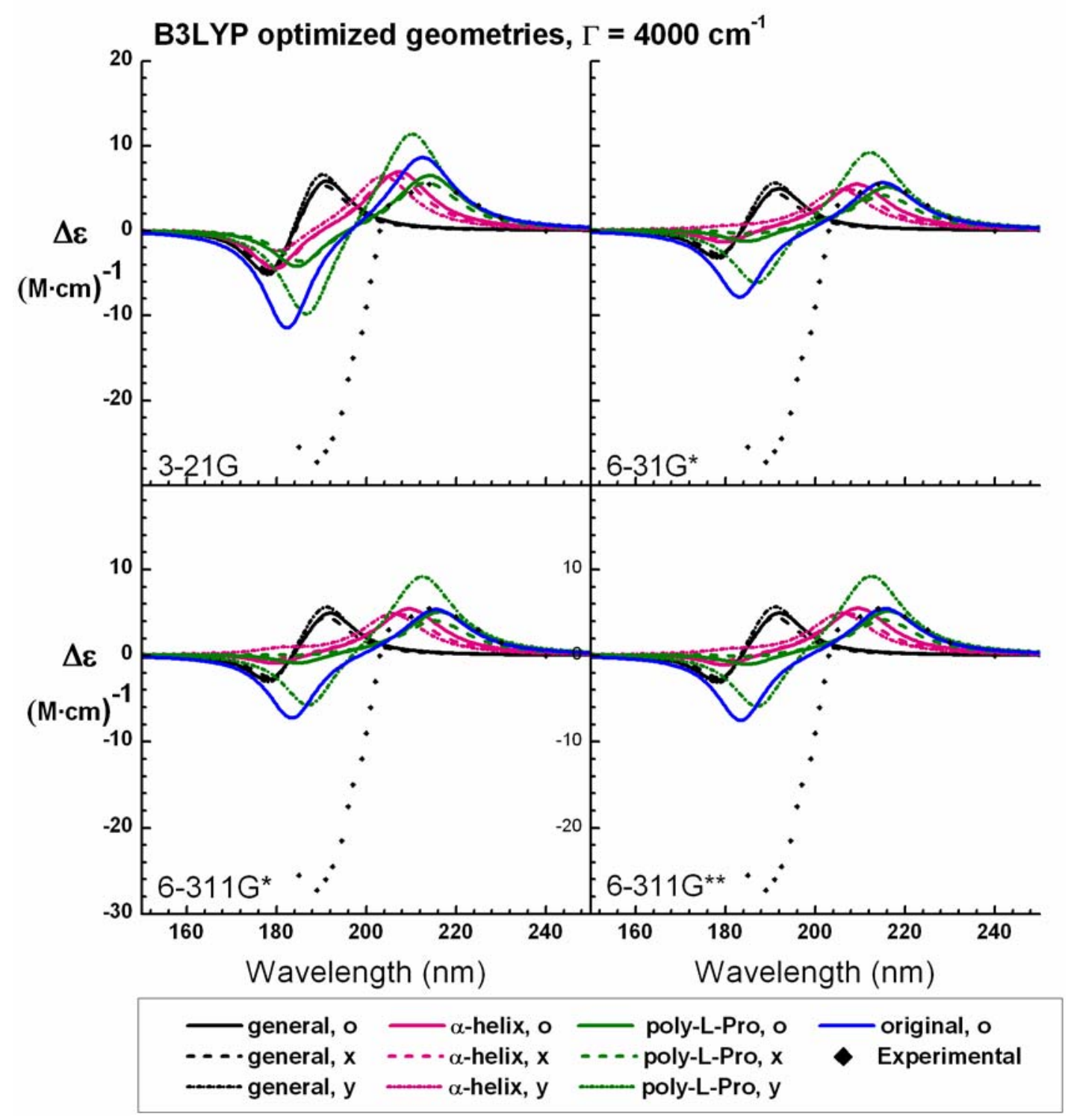

The experimental CD (in trifluoroethanol) was obtained from [Pancoska, P.; Fric, I.; Blaha, K. Coll. Czech.Chem. Comm. 1979, 44, 1296.] 
Supplement Figure 11. Cyclo(L-Pro-Gly) Calculated CD, BLYP Geometries

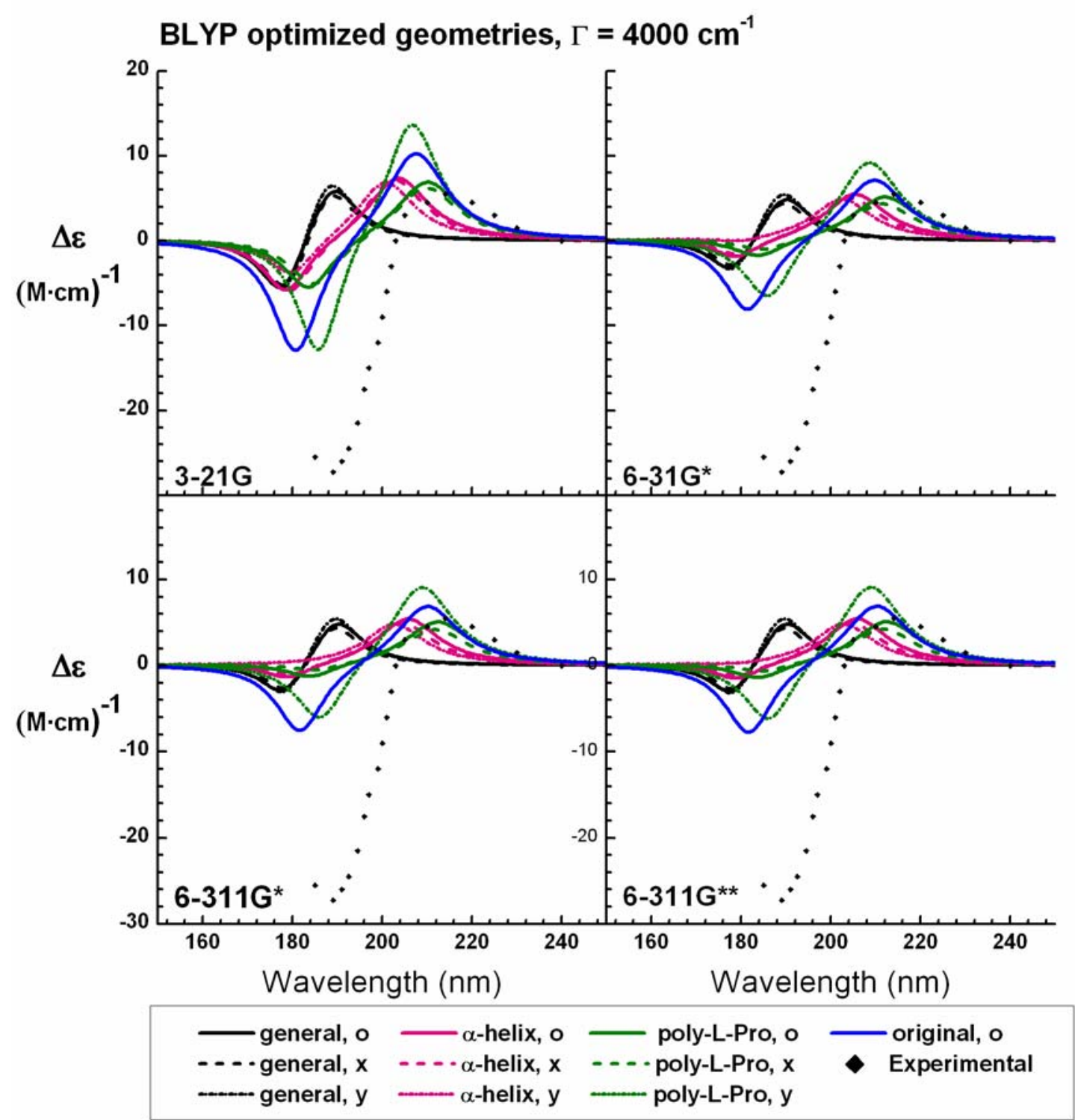

The experimental CD (in trifluoroethanol) was obtained from [Pancoska, P.; Fric, I.; Blaha, K. Coll. Czech.Chem. Comm. 1979, 44, 1296.] 
Supplement Figure 12. Cyclo(L-Pro-Gly) Calculated CD, BVWN Geometries

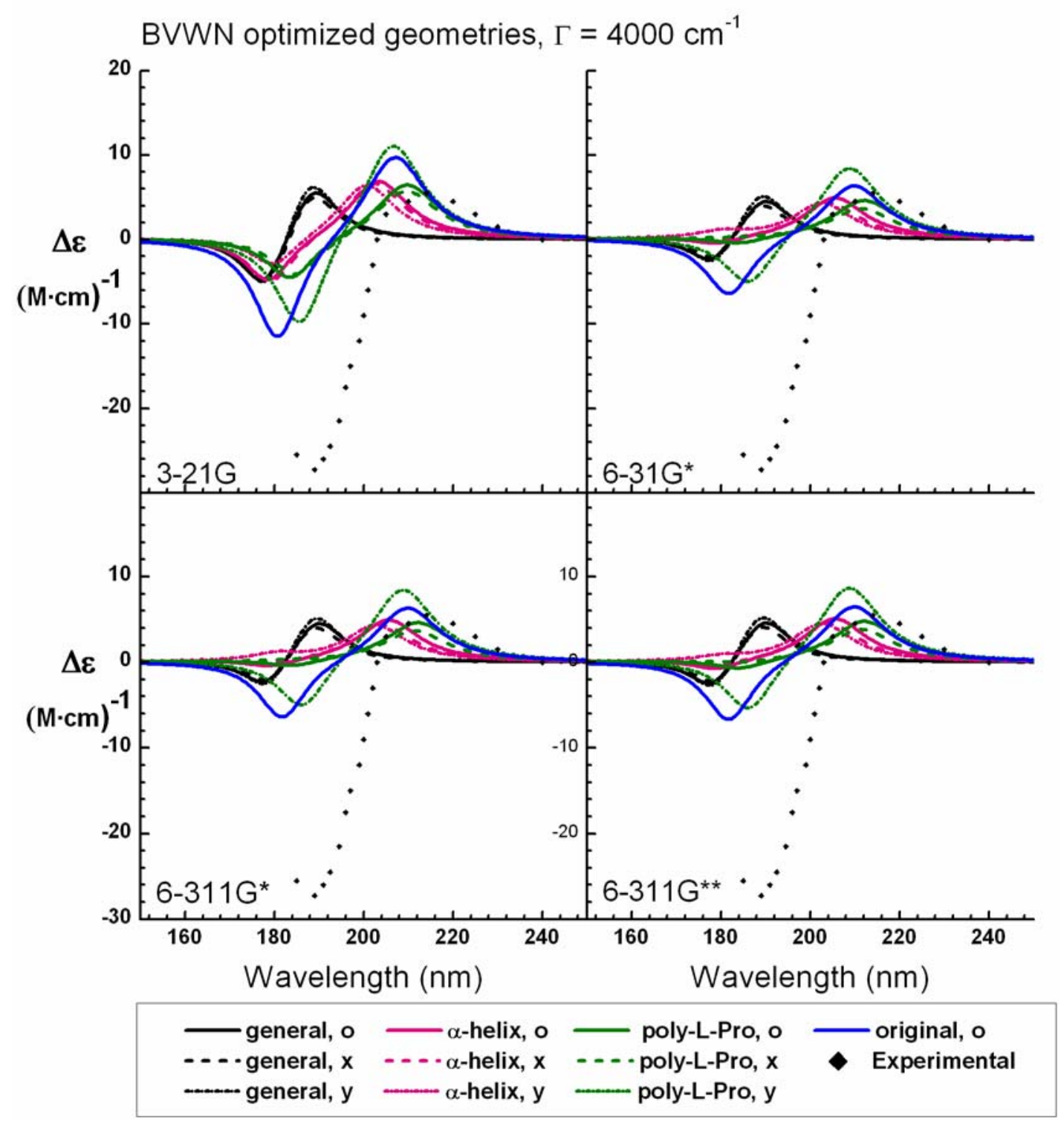

The experimental CD (in trifluoroethanol) was obtained from [Pancoska, P.; Fric, I.; Blaha, K. Coll. Czech.Chem. Comm. 1979, 44, 1296.] 
Supplement Figure 13. Cyclo(L-Pro-Gly) Calculated CD, MP2 Geometries

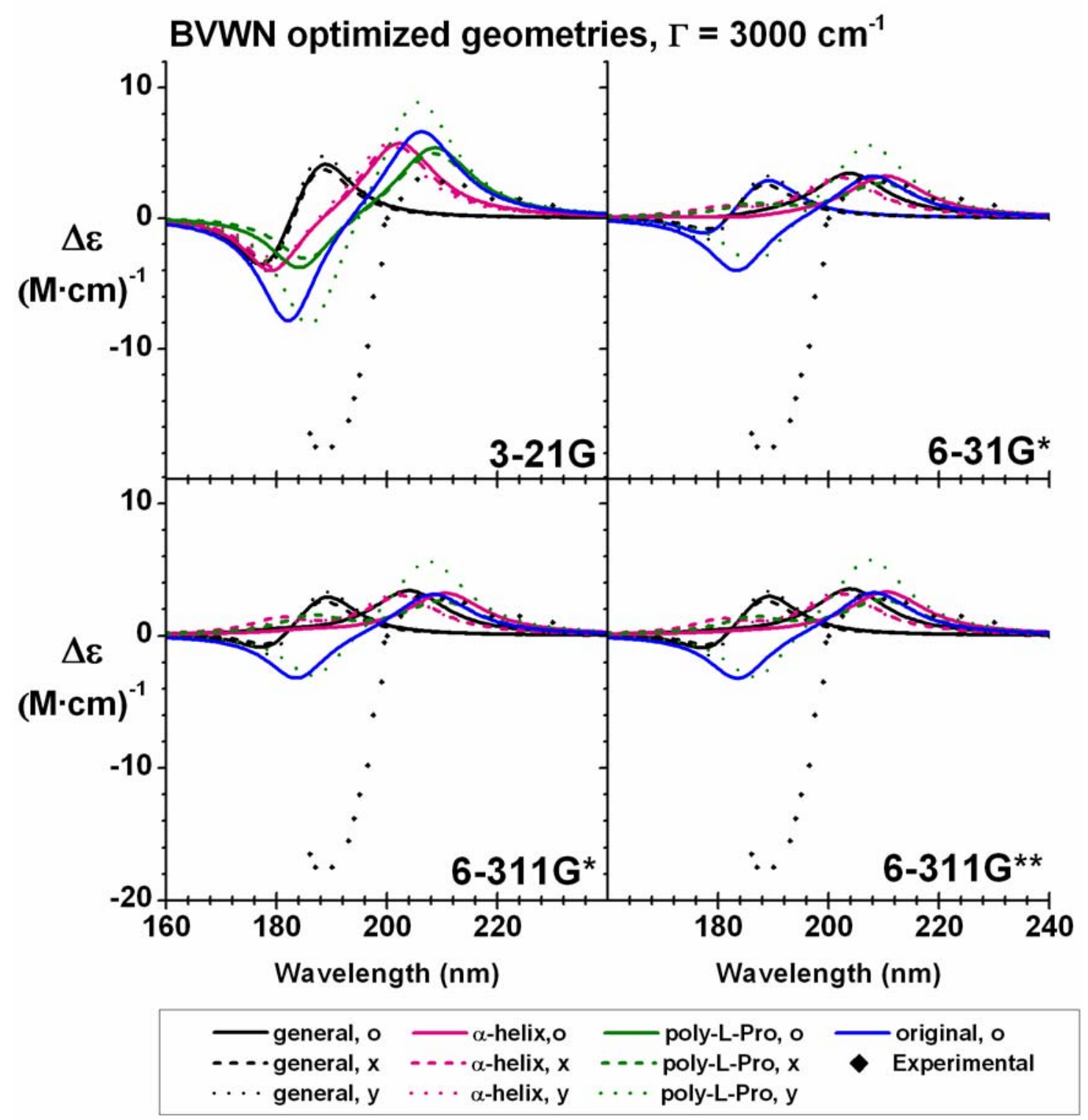

The experimental CD (in trifluoroethanol) was obtained from [Pancoska, P.; Fric, I.; Blaha, K. Coll. Czech.Chem. Comm. 1979, 44, 1296.] 
Supplement Figure 14. Cyclo(L-Pro-L-Ala) Calculated CD, CVFF Geometries

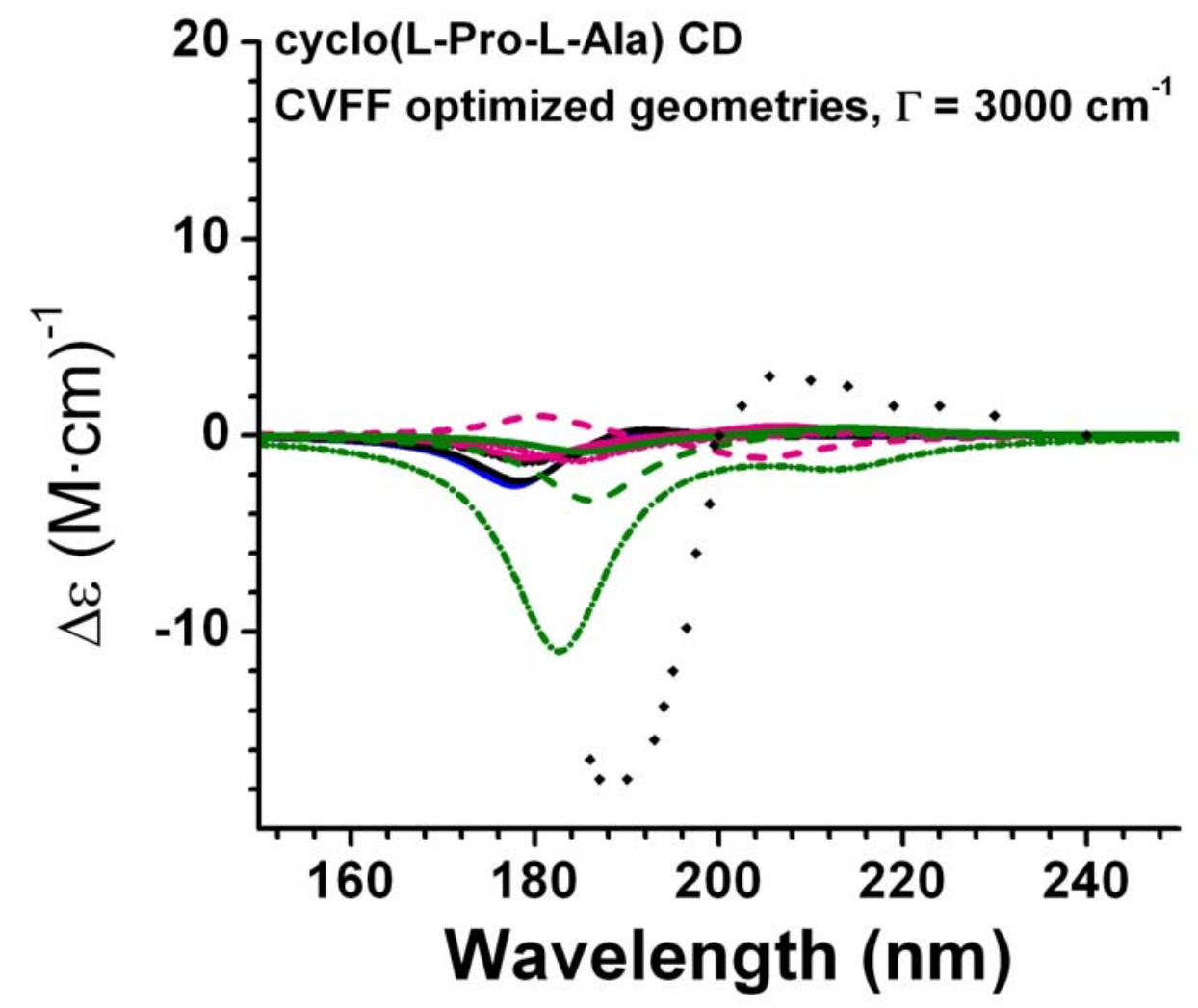

$$
\begin{aligned}
& \text { - general, } 0-\alpha \text {-helix,o poly-L-Pro, } 0-\text { original, } 0 \\
& \text { - - general, } x \quad--\alpha \text {-helix, } x \quad-\cdot \text { poly-L-Pro, } x \text { • Experimental } \\
& \text { general, y } \alpha \text {-helix, } y \quad \cdots \text { poly-L-Pro, } y
\end{aligned}
$$

The experimental CD (in trifluoroethanol) was obtained from [Pancoska, P.; Fric, I.; Blaha, K. Coll. Czech.Chem. Comm. 1979, 44, 1296.] 
Supplement Figure 15. Cyclo(L-Pro-L-Ala) Calculated CD, RHF Geometries

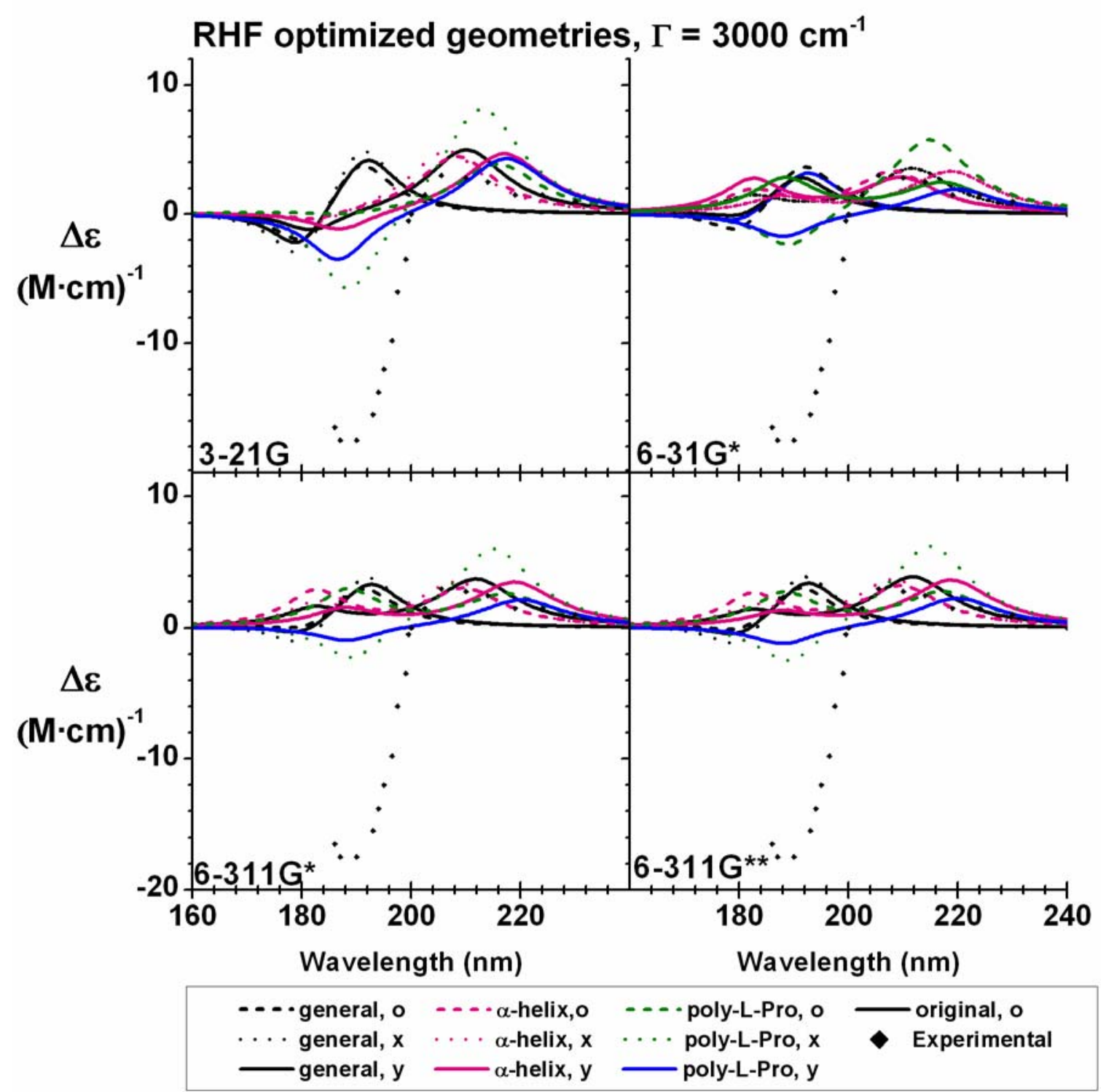

The experimental CD (in trifluoroethanol) was obtained from [Pancoska, P.; Fric, I.; Blaha, K. Coll. Czech.Chem. Comm. 1979, 44, 1296.] 
Supplement Figure 16. Cyclo(L-Pro-L-Ala) Calculated CD, B3LYP Geometries

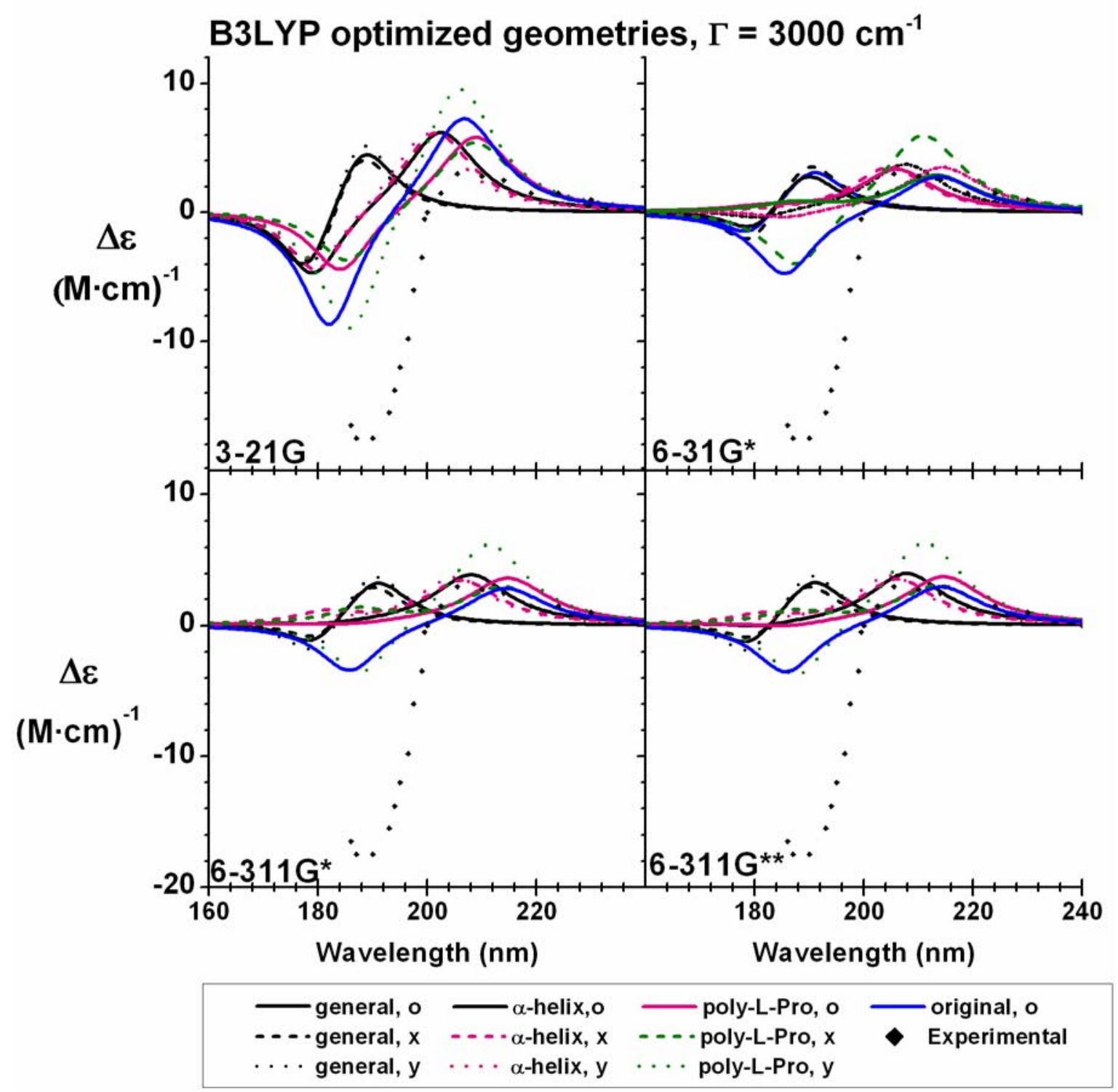

The experimental CD (in trifluoroethanol) was obtained from [Pancoska, P.; Fric, I.; Blaha, K. Coll. Czech.Chem. Comm. 1979, 44, 1296.] 
Supplement Figure 17. Cyclo(L-Pro-L-Ala) Calculated CD, BLYP Geometries

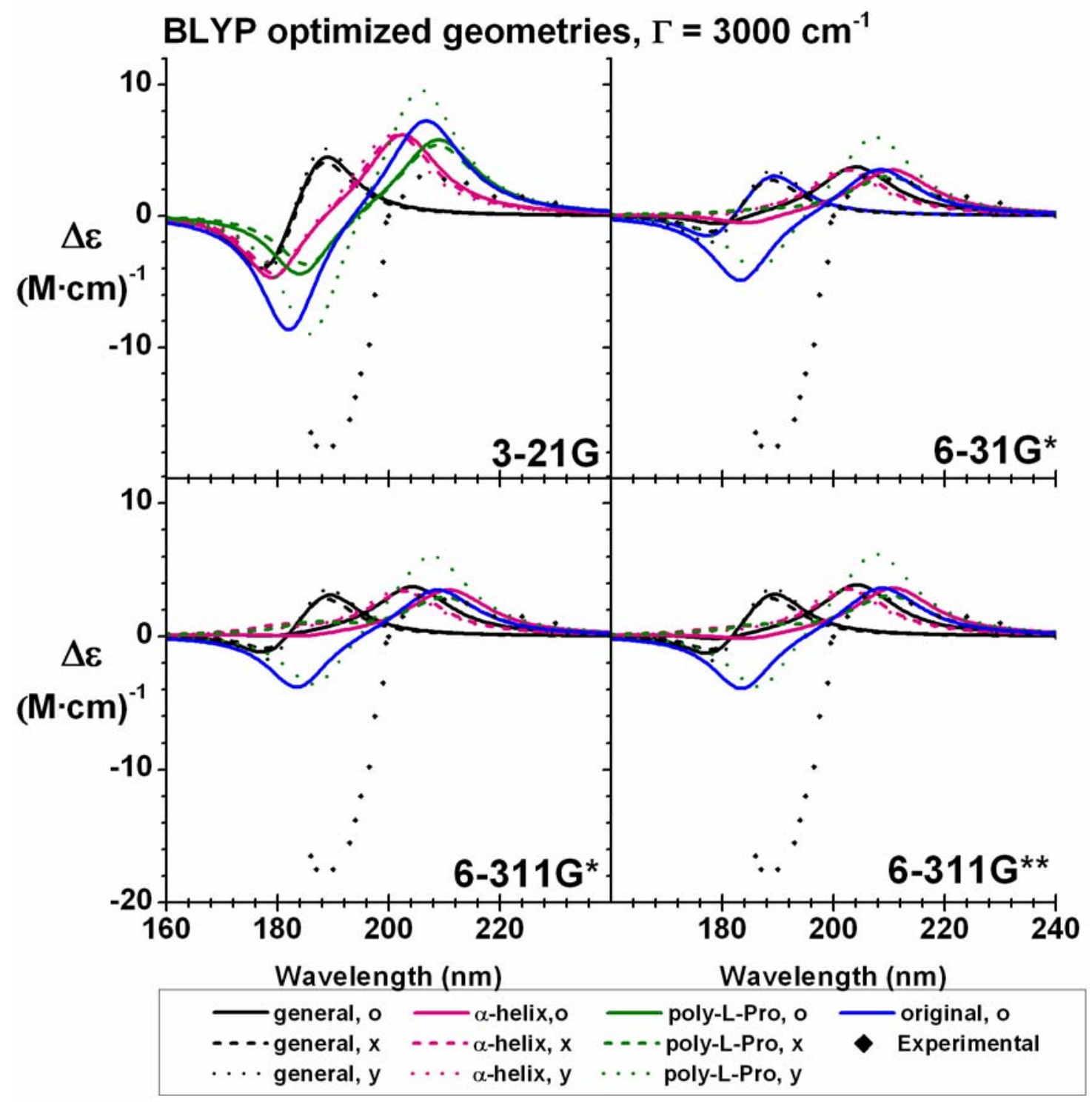

The experimental CD (in trifluoroethanol) was obtained from [Pancoska, P.; Fric, I.; Blaha, K. Coll. Czech.Chem. Comm. 1979, 44, 1296.] 
Supplement Figure 18. Cyclo(L-Pro-L-Ala) Calculated CD, BVWN Geometries

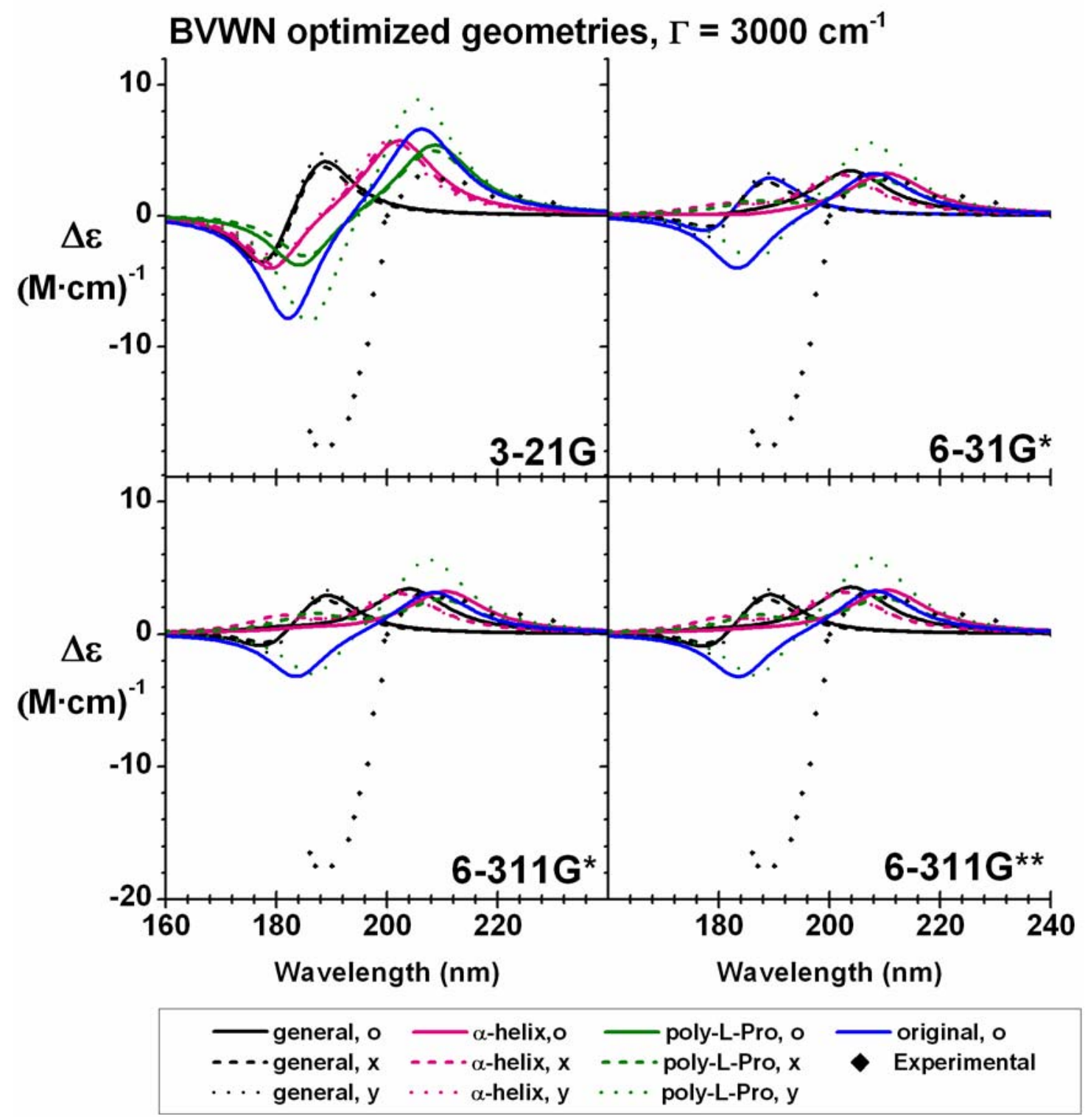

The experimental CD (in trifluoroethanol) was obtained from [Pancoska, P.; Fric, I.; Blaha, K. Coll. Czech.Chem. Comm. 1979, 44, 1296.] 
Supplement Figure 19. Cyclo(L-Pro-L-Ala) Calculated CD, MP2 Geometries

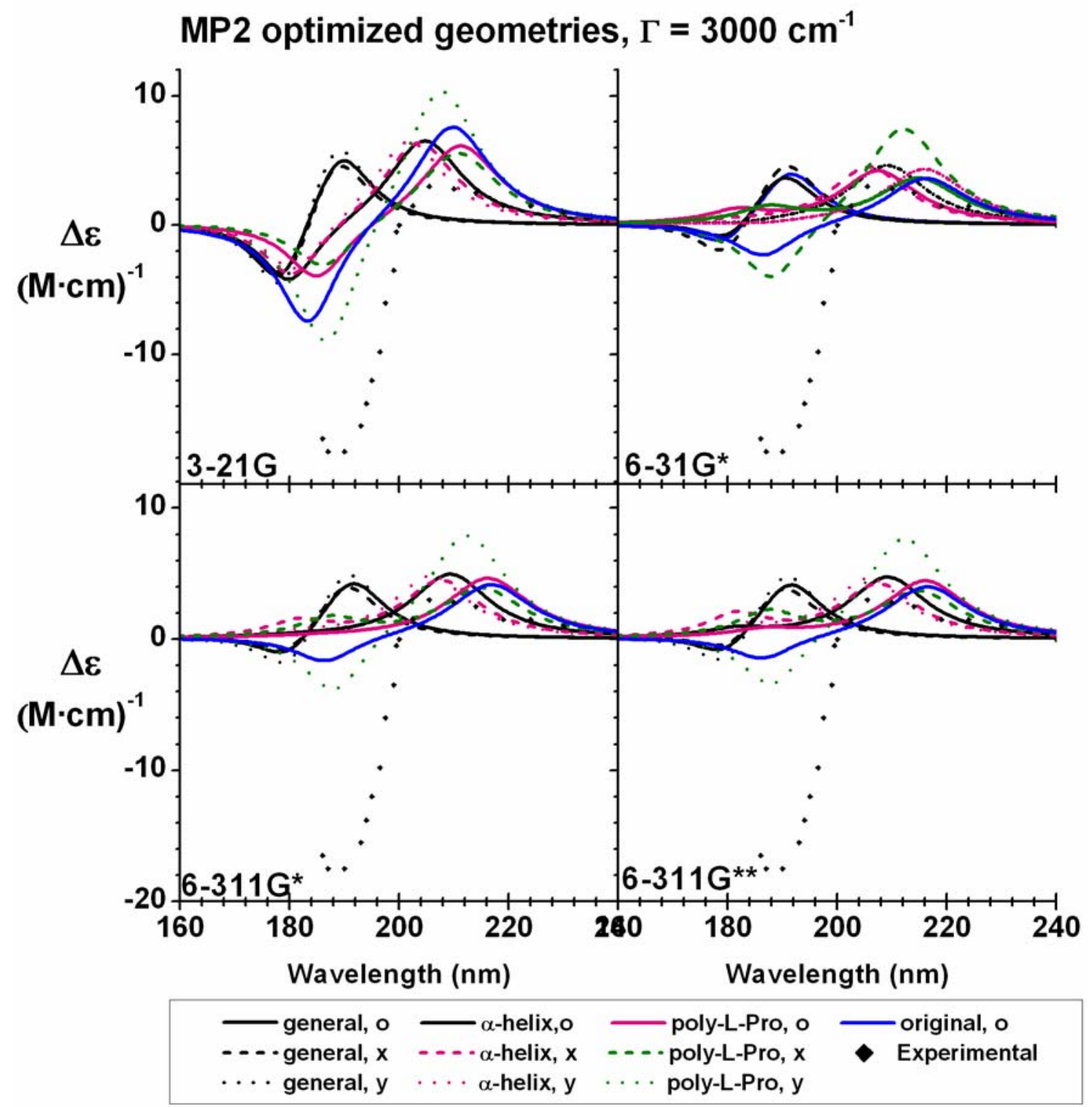

The experimental CD (in trifluoroethanol) was obtained from [Pancoska, P.; Fric, I.; Blaha, K. Coll. Czech.Chem. Comm. 1979, 44, 1296.] 
Supplement Figure 20. Cyclo(L-Pro-L-Leu) Calculated CD, CVFF Geometries

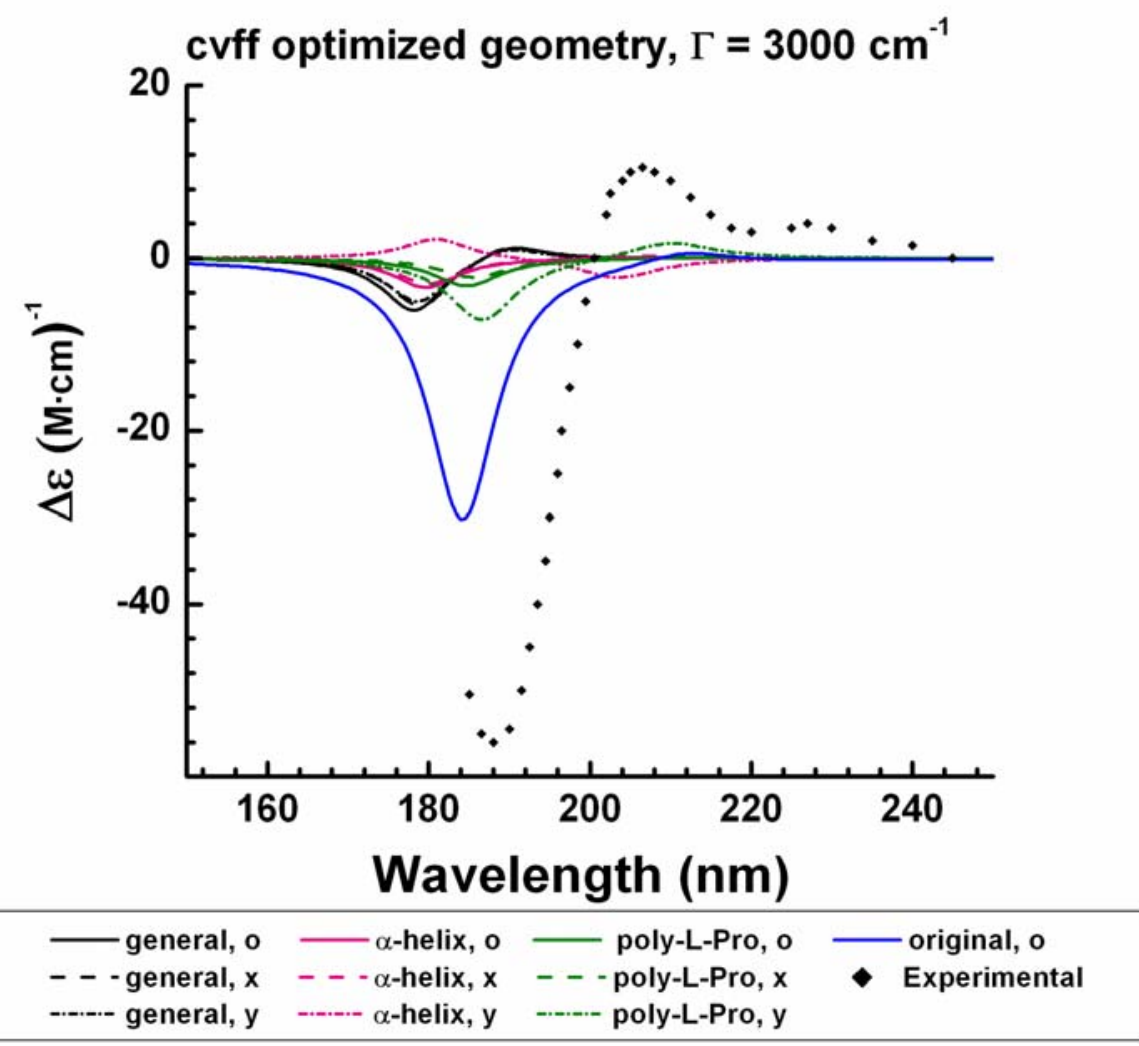

The experimental CD (in trifluoroethanol) was obtained from [Pancoska, P.; Fric, I.; Blaha, K. Coll. Czech.Chem. Comm. 1979, 44, 1296.] 
Supplement Figure 21. Cyclo(L-Pro-L-Leu) Calculated CD, RHF Geometries

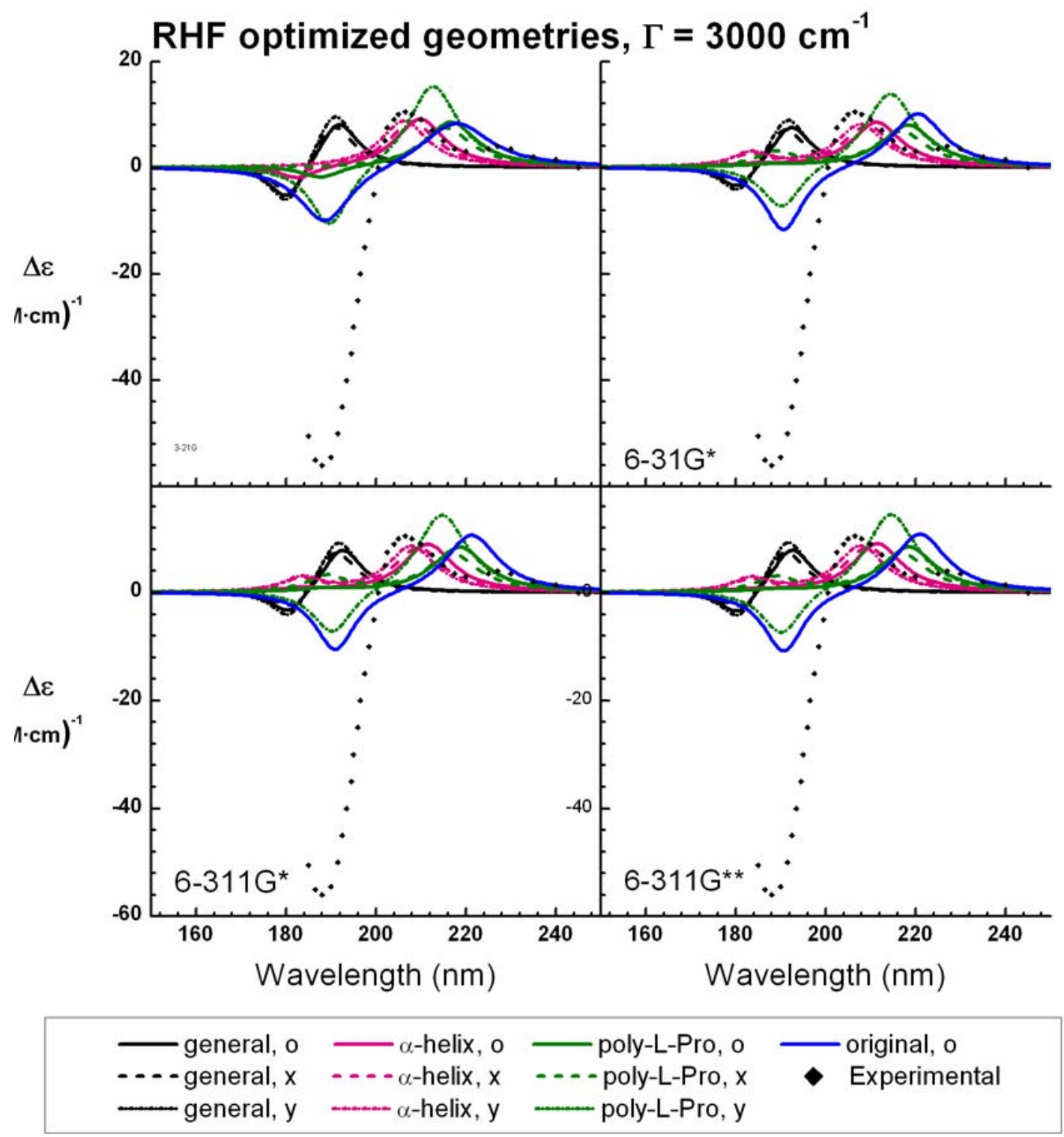

The experimental CD (in trifluoroethanol) was obtained from [Pancoska, P.; Fric, I.; Blaha, K. Coll. Czech.Chem. Comm. 1979, 44, 1296.] 
Supplement Figure 22. Cyclo(L-Pro-L-Leu) Calculated CD, B3LYP Geometries

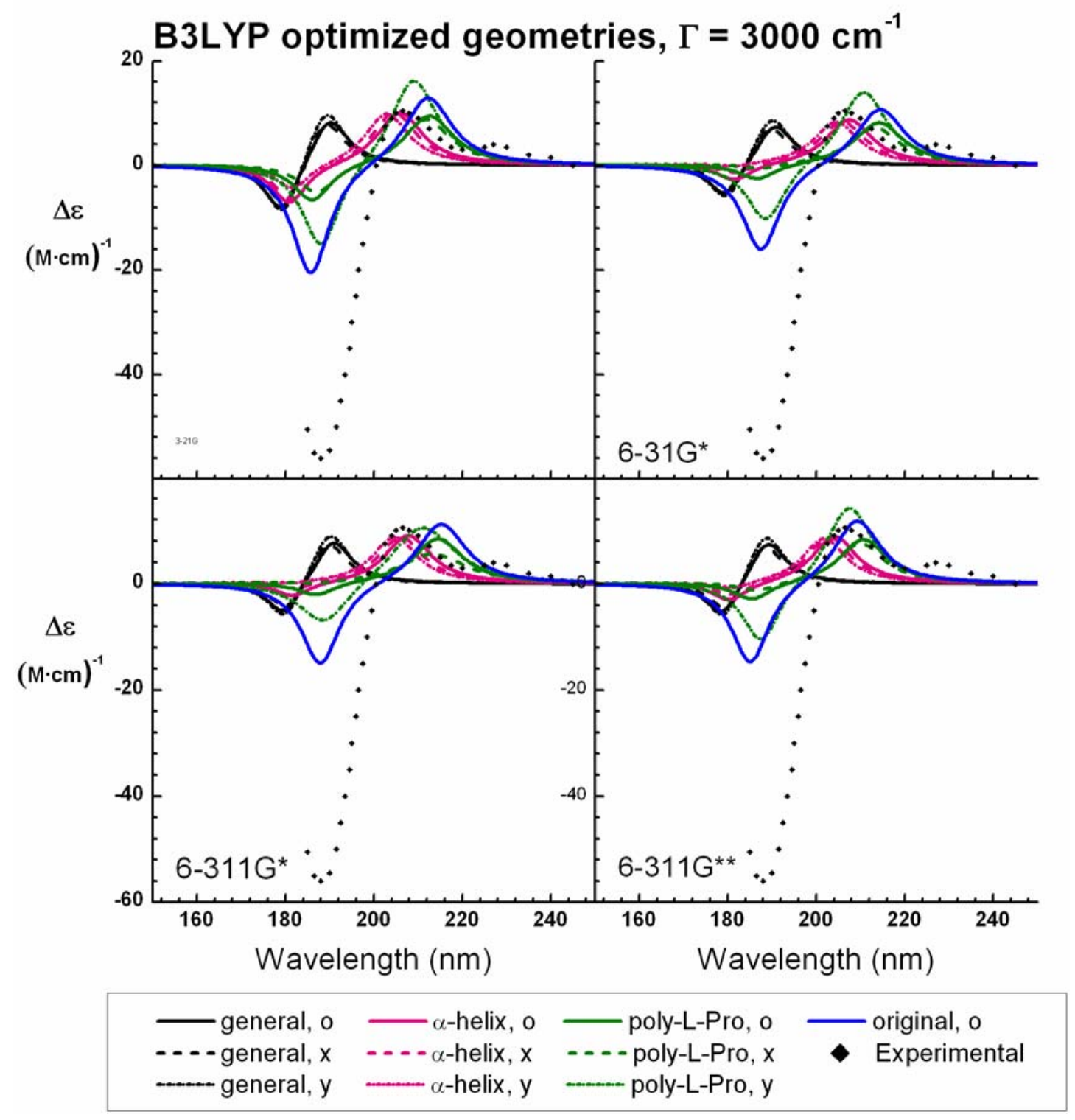

The experimental CD (in trifluoroethanol) was obtained from [Pancoska, P.; Fric, I.; Blaha, K. Coll. Czech.Chem. Comm. 1979, 44, 1296.] 
Supplement Figure 23. Cyclo(L-Pro-L-Leu) Calculated CD, BLYP Geometries

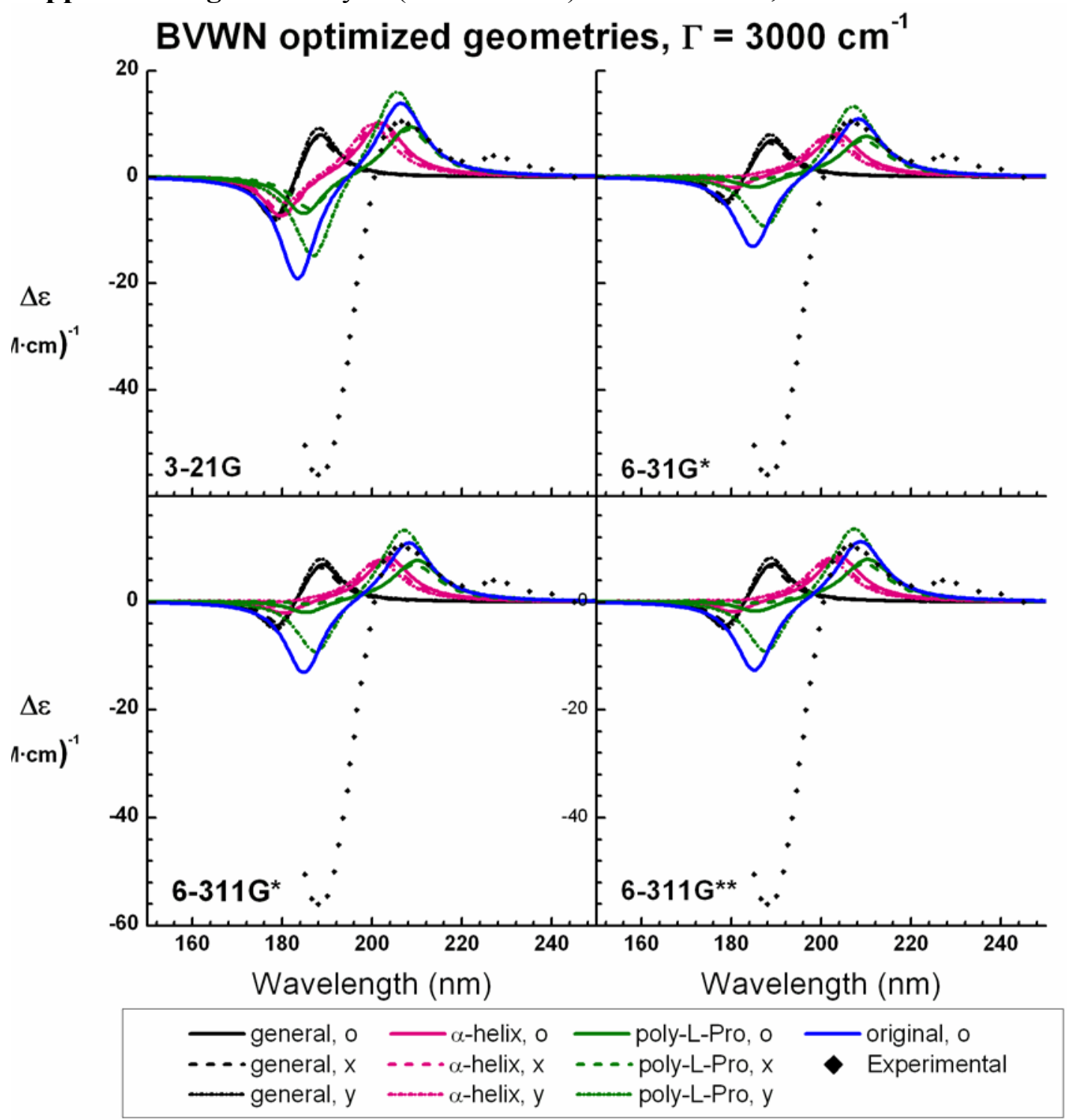

The experimental CD (in trifluoroethanol) was obtained from [Pancoska, P.; Fric, I.; Blaha, K. Coll. Czech.Chem. Comm. 1979, 44, 1296.] 
Supplement Figure 24. Cyclo(L-Pro-L-Leu) Calculated CD, BVWN Geometries

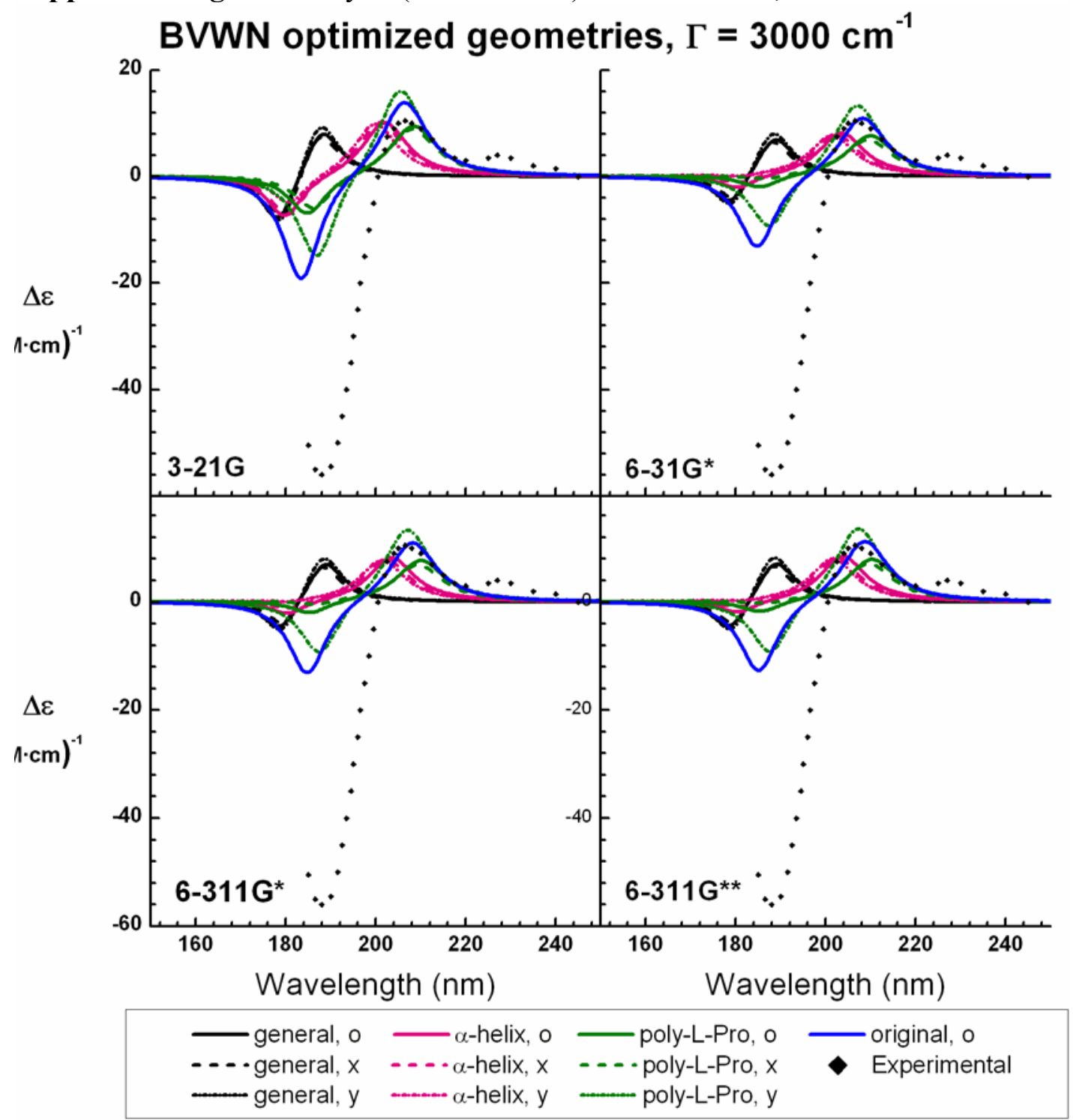

The experimental CD (in trifluoroethanol) was obtained from [Pancoska, P.; Fric, I.; Blaha, K. Coll. Czech.Chem. Comm. 1979, 44, 1296.] 
Supplement Figure 25. Cyclo(L-Pro-L-Leu) Calculated CD, MP2 Geometries

MP2 optimized geometries, $\Gamma=3000 \mathrm{~cm}^{-1}$

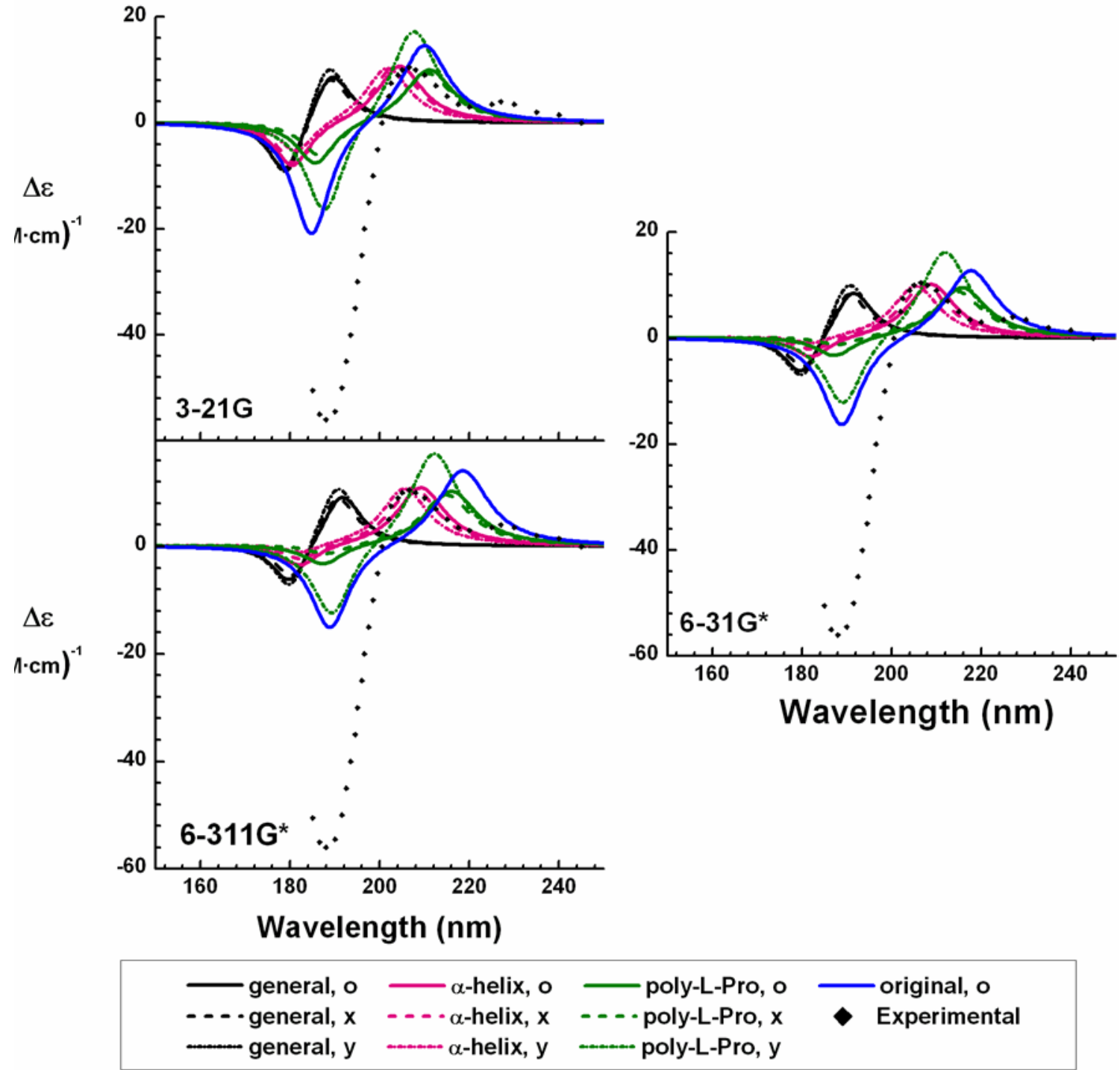

The experimental CD (in trifluoroethanol) was obtained from [Pancoska, P.; Fric, I.; Blaha, K. Coll. Czech.Chem. Comm. 1979, 44, 1296.] 
Supplement Table 1. Bond Lengths of Cyclo(L-Ala-L-Ala).

\begin{tabular}{|c|c|c|c|c|c|c|c|c|c|c|c|}
\hline & \multicolumn{6}{|c|}{ Diketopiperazine Ring } & \multicolumn{2}{|c|}{ Carbonyl } & \multicolumn{2}{|c|}{ Side Chains } \\
\hline & & $\begin{array}{l}\bar{Z} \\
\bar{u}\end{array}$ & $\begin{array}{l}8 \\
U_{1}^{\prime} \\
\frac{1}{Z}\end{array}$ & $\begin{array}{l}\bar{U} \\
\delta^{\prime} \\
U^{\prime}\end{array}$ & $\begin{array}{l}z_{1}^{2} \\
\bar{u}\end{array}$ & 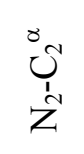 & $\begin{array}{l}\mho^{\prime} \\
\mho^{\prime} \\
\mho^{\prime}\end{array}$ & $\begin{array}{l}0_{-1}^{-} \\
J^{\prime}-\end{array}$ & $\begin{array}{l}0_{-1}^{N} \\
U^{\prime}\end{array}$ & $\begin{array}{l}\underbrace{\infty} \\
\mho^{\prime} \\
ن\end{array}$ & $\begin{array}{l}\infty \\
\bigcup_{0}^{\prime} \\
\sigma^{\prime}\end{array}$ \\
\hline Crystal $^{\mathrm{a}}$ & II & 1.33 & 1.45 & 1.52 & 1.33 & 1.46 & 1.52 & 1.24 & 1.24 & 1.52 & 1.52 \\
\hline CVFF & $\mathrm{I}$ & 1.36 & 1.48 & 1.54 & 1.36 & 1.48 & 1.54 & 1.24 & 1.24 & 1.53 & 1.53 \\
\hline CVFF & II & 1.33 & 1.49 & 1.57 & 1.33 & 1.49 & 1.57 & 1.23 & 1.23 & 1.54 & 1.54 \\
\hline \multicolumn{12}{|c|}{ 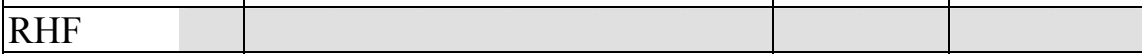 } \\
\hline $3-21 G$ & II & 1.33 & 1.43 & 1.52 & 1.35 & 1.46 & 1.52 & 1.24 & 1.24 & 1.53 & 1.53 \\
\hline $6-31 G^{*}$ & II & 1.35 & 1.45 & 1.52 & 1.35 & 1.45 & 1.52 & 1.20 & 1.20 & 1.52 & 1.52 \\
\hline $6-311 \mathrm{G}^{*}$ & $\mathrm{I}$ & 1.35 & 1.45 & 1.52 & 1.35 & 1.45 & 1.52 & 1.20 & 1.20 & 1.53 & 1.53 \\
\hline $6-311 G^{* *}$ & $\mathrm{I}$ & 1.35 & 1.45 & 1.52 & 1.35 & 1.45 & 1.52 & 1.20 & 1.20 & 1.53 & 1.53 \\
\hline \multicolumn{12}{|c|}{\begin{tabular}{|l|l|l|l|} 
B3LYP & & \\
\end{tabular}} \\
\hline $3-21 G$ & II & 1.32 & 1.48 & 1.54 & 1.36 & 1.48 & 1.54 & 1.24 & 1.24 & 1.53 & 1.53 \\
\hline $6-31 G^{*}$ & II & 1.36 & 1.46 & 1.54 & 1.36 & 1.46 & 1.54 & 1.22 & 1.22 & 1.53 & 1.53 \\
\hline $6-311 G^{*}$ & II & 1.36 & 1.46 & 1.53 & 1.36 & 1.46 & 1.53 & 1.22 & 1.22 & 1.53 & 1.53 \\
\hline $6-311 G^{* *}$ & II & 1.36 & 1.46 & 1.53 & 1.36 & 1.44 & 1.53 & 1.22 & 1.22 & 1.53 & 1.53 \\
\hline \multicolumn{12}{|l|}{ BLYP } \\
\hline $3-21 \mathrm{G}$ & II & 1.38 & 1.49 & 1.56 & 1.38 & 1.49 & 1.56 & \begin{tabular}{|l|}
1.25 \\
\end{tabular} & 1.25 & 1.55 & 1.55 \\
\hline $6-31 G^{*}$ & II & 1.37 & 1.48 & 1.55 & 1.37 & 1.48 & 1.55 & 1.24 & 1.24 & 1.54 & 1.54 \\
\hline $6-311 G^{*}$ & II & 1.37 & 1.48 & 1.55 & 1.37 & 1.48 & 1.55 & 1.23 & 1.23 & 1.54 & 1.54 \\
\hline $6-311 G^{* *}$ & II & 1.37 & 1.48 & 1.55 & 1.37 & 1.48 & 1.55 & 1.23 & 1.23 & 1.54 & 1.54 \\
\hline \multicolumn{12}{|l|}{ BVWN } \\
\hline $3-21 G$ & II & 1.38 & 1.49 & 1.56 & 1.38 & 1.49 & 1.56 & 1.25 & 1.26 & 1.55 & 1.55 \\
\hline $6-31 G^{*}$ & II & 1.38 & 1.48 & 1.55 & 1.38 & 1.48 & 1.55 & 1.24 & 1.24 & 1.55 & 1.55 \\
\hline $6-311 \mathrm{G}^{*}$ & II & 1.37 & 1.48 & 1.55 & 1.37 & 1.48 & 1.55 & 1.23 & 1.23 & 1.55 & 1.55 \\
\hline $6-311 G^{* *}$ & II & 1.37 & 1.48 & 1.54 & 1.37 & 1.48 & 1.54 & 1.23 & 1.23 & 1.55 & 1.55 \\
\hline \multicolumn{12}{|l|}{ MP2 } \\
\hline $3-21 G$ & I & 1.37 & 1.48 & 1.54 & 1.37 & 1.48 & 1.54 & 1.25 & 1.25 & 1.55 & 1.55 \\
\hline $6-31 G^{*}$ & I & 1.36 & 1.46 & 1.52 & 1.36 & 1.46 & 1.52 & 1.23 & 1.23 & 1.53 & 1.53 \\
\hline $6-311 G^{*}$ & I & 1.36 & 1.46 & 1.52 & 1.36 & 1.46 & 1.52 & 1.22 & 1.22 & 1.53 & 1.53 \\
\hline $6-311 \mathrm{G}^{* *}$ & I & 1.36 & 1.46 & 1.52 & 1.36 & 1.46 & 1.52 & 1.22 & 1.22 & 1.53 & 1.53 \\
\hline \multicolumn{12}{|l|}{ MP2 } \\
\hline $3-21 G$ & II & 1.37 & 1.48 & 1.54 & 1.37 & 1.48 & 1.54 & 1.25 & 1.25 & 1.55 & 1.55 \\
\hline $6-31 G^{*}$ & I & 1.36 & 1.46 & 1.52 & 1.36 & 1.47 & 1.52 & 1.23 & 1.23 & 1.53 & 1.53 \\
\hline $6-311 G^{*}$ & II & 1.36 & 1.46 & 1.52 & 1.36 & 1.46 & 1.52 & 1.22 & 1.22 & 1.53 & 1.53 \\
\hline $6-311 G^{* *}$ & II & 1.36 & 1.46 & 1.52 & 1.36 & 1.46 & 1.52 & 1.22 & 1.22 & 1.53 & 1.53 \\
\hline
\end{tabular}

All bond lengths are in $\AA$. ${ }^{\text {a }}$ Crystal structure obtained from [Sletten, E. J. Am. Chem. Soc. 1970, 92, 172.] 
Supplement Table 2. Bond Angles of Cyclo(L-Ala-L-Ala).

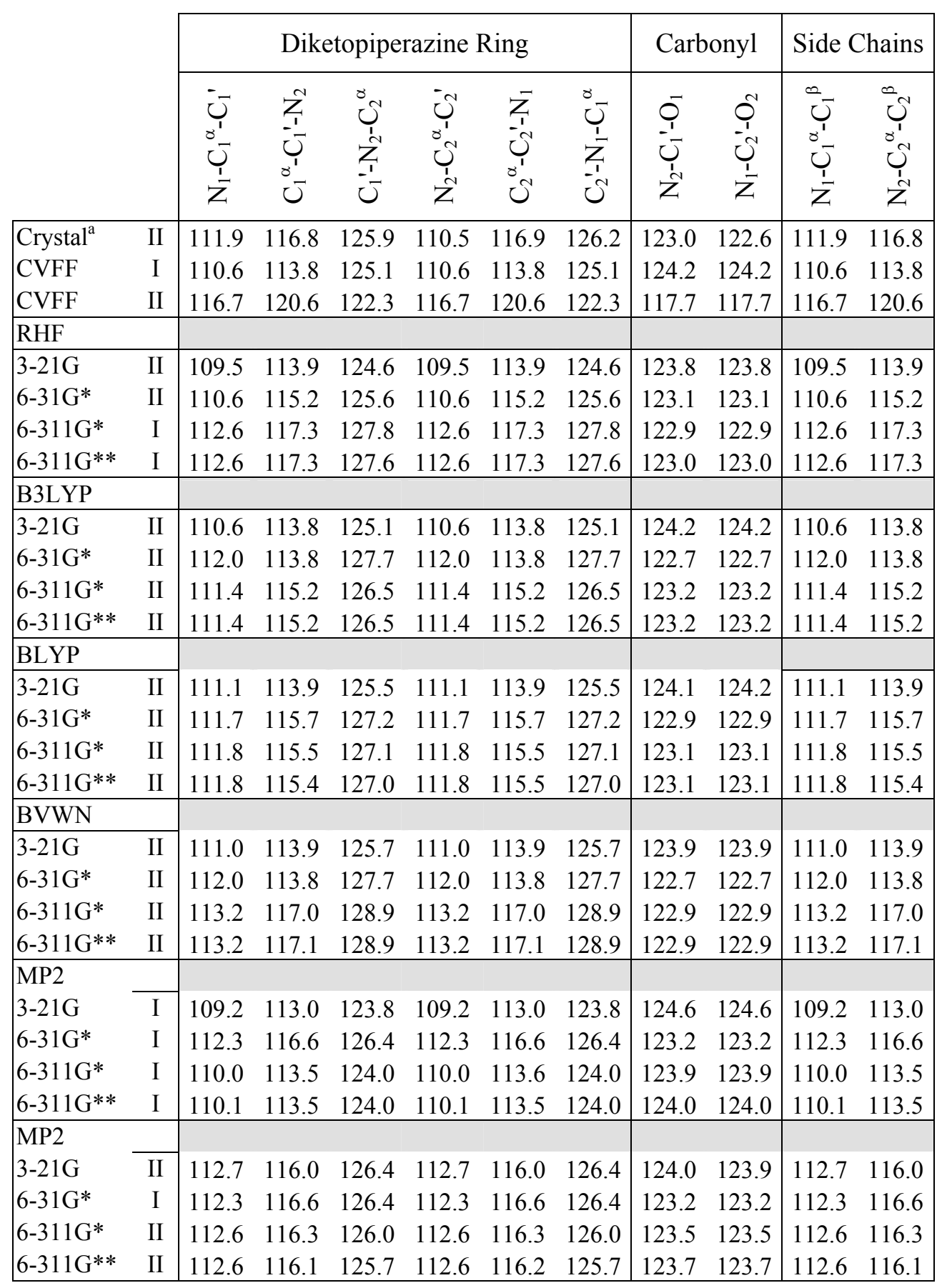

All angles are in degrees. ${ }^{a}$ Crystal structure obtained from [Sletten, E. J. Am. Chem. Soc. 1970, 92, 172.] 
Supplement Table 3. Bond Dihedral Angles of Cyclo(L-Ala-L-Ala).

\begin{tabular}{|c|c|c|c|c|c|c|c|c|c|}
\hline & & $\phi_{1}$ & $\psi_{1}$ & $\omega_{1}$ & $\phi_{2}$ & $\psi_{2}$ & $\omega_{2}$ & $\chi_{1}^{1}$ & $\chi_{2}^{1}$ \\
\hline Crystal $^{\mathrm{a}}$ & II & -25.6 & 20.6 & 8.1 & -32.5 & 26.8 & 0.8 & -25.6 & 20.6 \\
\hline CVFF & I & -37.1 & 33.9 & 1.9 & -37.1 & 33.9 & 1.9 & -37.1 & 33.9 \\
\hline CVFF & II & 6.7 & -6.4 & -0.3 & 6.7 & -6.4 & -0.4 & 6.7 & -6.4 \\
\hline \multicolumn{10}{|l|}{ RHF } \\
\hline $3-21 \mathrm{G}$ & II & -40.1 & 35.4 & 3.2 & -40.0 & 35.3 & 3.3 & -40.1 & 35.4 \\
\hline $6-31 G^{*}$ & II & -37.3 & 25.0 & 11.0 & -37.3 & 25.0 & 11.0 & -37.3 & 25.0 \\
\hline $6-311 G^{*}$ & I & 19.7 & -12.0 & -7.2 & 19.7 & -12.0 & -7.2 & 19.7 & -12.0 \\
\hline $6-311 \mathrm{G}^{* *}$ & I & 20.9 & -12.0 & -8.3 & 20.9 & -12.0 & -8.3 & 20.9 & -12.0 \\
\hline \multicolumn{10}{|l|}{\begin{tabular}{|l} 
B3LYP \\
\end{tabular}} \\
\hline $3-21 \mathrm{G}$ & II & -37.1 & 33.9 & 1.9 & -37.1 & 33.9 & 1.9 & -37.1 & 33.9 \\
\hline $6-31 G^{*}$ & II & -32.3 & 22.1 & 9.0 & -32.3 & 22.1 & 9.0 & -32.3 & 22.1 \\
\hline $6-311 G^{*}$ & II & -33.4 & 23.2 & 9.0 & -33.4 & 23.2 & 9.0 & -33.4 & 23.2 \\
\hline $6-311 G^{* *}$ & II & -33.4 & 23.5 & 8.7 & -33.4 & 23.5 & 8.7 & -33.4 & 23.5 \\
\hline \multicolumn{10}{|l|}{ BLYP } \\
\hline $3-21 G$ & II & -35.3 & 32.3 & 1.6 & -35.2 & 32.4 & 1.6 & -35.3 & 32.3 \\
\hline $6-31 G^{*}$ & II & -30.2 & 19.8 & 9.3 & -30.2 & 19.8 & 9.3 & -30.2 & 19.8 \\
\hline $6-311 \mathrm{G}^{*}$ & II & -30.8 & 20.4 & 9.3 & -30.8 & 20.4 & 9.3 & -30.8 & 20.4 \\
\hline $6-311 \mathrm{G}^{* *}$ & II & -30.9 & 20.9 & 8.9 & -30.9 & 21.0 & 8.9 & -30.9 & 20.9 \\
\hline \multicolumn{10}{|l|}{ BVWN } \\
\hline $3-21 G$ & II & -35.0 & 32.2 & 1.5 & -35.0 & 32.2 & 1.5 & -35.0 & 32.2 \\
\hline $6-31 G^{*}$ & II & -27.5 & 16.8 & 9.8 & -27.5 & 16.8 & 9.8 & -27.5 & 16.8 \\
\hline $6-311 G^{*}$ & II & 12.7 & -8.0 & -4.4 & 12.7 & -8.0 & -4.4 & 12.7 & -8.0 \\
\hline $6-311 \mathrm{G}^{* *}$ & II & 12.2 & -7.3 & -4.5 & 12.2 & -7.3 & -4.5 & 12.2 & -7.3 \\
\hline \multicolumn{10}{|l|}{ MP2 } \\
\hline $3-21 \mathrm{G}$ & I & -43.0 & 38.2 & 3.2 & -43.0 & 38.2 & 3.2 & -43.0 & 38.2 \\
\hline $6-31 G^{*}$ & I & 29.2 & -13.9 & -14.5 & 29.2 & -13.9 & -14.4 & 29.2 & -13.9 \\
\hline $6-311 \mathrm{G}^{*}$ & I & -44.1 & 31.6 & 10.7 & -44.1 & 31.6 & 10.7 & -44.1 & 31.6 \\
\hline $6-311 \mathrm{G}^{* *}$ & I & -44.7 & 30.1 & 12.8 & -44.7 & 30.1 & 12.8 & -44.7 & 30.1 \\
\hline \multicolumn{10}{|l|}{ MP2 } \\
\hline $3-21 G$ & II & -18.3 & 28.8 & -9.5 & -18.3 & 28.8 & -9.5 & -18.3 & 28.8 \\
\hline $6-31 \mathrm{G}^{*}$ & I & -13.9 & 29.2 & -14.5 & -13.9 & 29.2 & -14.5 & -13.9 & 29.2 \\
\hline $6-311 G^{*}$ & II & -14.3 & 30.2 & -15.0 & -14.3 & 30.2 & -15.1 & -14.3 & 30.2 \\
\hline $6-311 G^{* *}$ & II & -13.4 & 31.5 & -17.2 & -13.4 & 31.5 & -17.2 & -13.4 & 31.5 \\
\hline
\end{tabular}

All angles are in degrees. ${ }^{a}$ Crystal structure obtained from [Sletten, E. J. Am. Chem. Soc. 1970, 92, 172.] 
Supplement Table 4. Bond Lengths of Cyclo(L-Pro-Gly).

\begin{tabular}{|c|c|c|c|c|c|c|c|c|c|c|c|c|}
\hline & \multicolumn{6}{|c|}{ Diketopiperazine Ring } & \multicolumn{2}{|c|}{ Carbonyl } & \multicolumn{4}{|c|}{ Proline Side Chain } \\
\hline & $\begin{array}{l}\bar{Z}_{1}^{\prime} \\
u^{\prime}\end{array}$ & 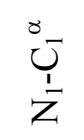 & $\begin{array}{l}\bigcup_{1}^{-} \\
\overbrace{}^{\prime}\end{array}$ & $\frac{7}{i}$ & $\begin{array}{l}U_{1}^{N} \\
Z^{N}\end{array}$ & $\begin{array}{l}\bigcup_{1}^{N} \\
\underbrace{\prime} \\
\mho^{\prime}\end{array}$ & $\begin{array}{l}0 \\
-1 \\
\dot{U}\end{array}$ & $\begin{array}{c}0^{N} \\
U^{-1}\end{array}$ & $\begin{array}{l}\infty \\
U_{1}^{\prime} \\
0^{\prime} \\
U\end{array}$ & $\begin{array}{l}U^{\prime} \\
\infty^{\prime} \\
U\end{array}$ & $\begin{array}{l}U_{1}^{\infty} \\
\check{U}^{\prime}\end{array}$ & $\begin{array}{l}\bar{Z}_{1} \\
\mathcal{U}^{\prime}\end{array}$ \\
\hline$\overline{\text { Crystal }^{\mathrm{a}}}$ & 1.33 & 1.46 & 1.52 & 1.34 & 1.46 & 1.52 & 1.23 & 1.23 & 1.52 & 1.53 & 1.52 & 1.46 \\
\hline CVFF & 1.36 & 1.49 & 1.58 & 1.34 & 1.50 & 1.57 & 1.23 & 1.23 & 1.55 & 1.54 & 1.53 & 1.47 \\
\hline \multicolumn{13}{|l|}{ RHF } \\
\hline $3-21 G$ & 1.35 & 1.47 & 1.52 & 1.35 & 1.46 & 1.52 & 1.22 & 1.22 & 1.54 & 1.55 & 1.54 & 1.47 \\
\hline $6-31 G^{*}$ & 1.35 & 1.46 & 1.52 & 1.35 & 1.45 & 1.52 & 1.12 & 1.12 & 1.54 & 1.54 & 1.53 & 1.46 \\
\hline $6-311 G^{*}$ & 1.34 & 1.46 & 1.52 & 1.35 & 1.45 & 1.52 & 1.19 & 1.20 & 1.54 & 1.53 & 1.53 & 1.45 \\
\hline $6-311 G^{* *}$ & 1.34 & 1.46 & 1.52 & 1.35 & 1.45 & 1.52 & 1.19 & 1.19 & 1.54 & 1.53 & 1.53 & 1.46 \\
\hline \multicolumn{13}{|l|}{ B3LYP } \\
\hline $3-21 \mathrm{G}$ & 1.36 & 1.49 & 1.54 & 1.37 & 1.48 & 1.54 & 1.24 & 1.24 & 1.55 & 1.56 & 1.55 & 1.48 \\
\hline $6-31 \mathrm{G}^{*}$ & 1.36 & 1.47 & 1.54 & 1.36 & 1.46 & 1.53 & 1.22 & 1.23 & 1.55 & 1.54 & 1.54 & 1.46 \\
\hline $6-311 G^{*}$ & 1.36 & 1.47 & 1.54 & 1.36 & 1.46 & 1.53 & 1.22 & 1.22 & 1.54 & 1.54 & 1.53 & 1.46 \\
\hline $6-311 \mathrm{G}^{* *}$ & 1.36 & 1.47 & 1.54 & 1.36 & 1.46 & 1.53 & 1.22 & 1.22 & 1.54 & 1.54 & 1.53 & 1.46 \\
\hline \multicolumn{13}{|l|}{ BLYP } \\
\hline $3-21 G$ & 1.38 & 1.50 & 1.54 & 1.38 & 1.49 & 1.56 & 1.25 & 1.25 & 1.56 & 1.57 & 1.56 & 1.50 \\
\hline $6-31 G^{*}$ & 1.37 & 1.49 & 1.54 & 1.38 & 1.47 & 1.55 & 1.24 & 1.24 & 1.56 & 1.55 & 1.55 & 1.48 \\
\hline $6-311 G^{*}$ & 1.37 & 1.49 & 1.54 & 1.37 & 1.47 & 1.55 & 1.23 & 1.23 & 1.56 & 1.55 & 1.55 & 1.48 \\
\hline $6-311 \mathrm{G}^{* *}$ & 1.37 & 1.49 & 1.55 & 1.37 & 1.47 & 1.55 & 1.23 & 1.23 & 1.56 & 1.55 & 1.55 & 1.48 \\
\hline \multicolumn{13}{|l|}{ BVWN } \\
\hline $3-21 \mathrm{G}$ & .38 & 1.51 & 1.56 & 1.38 & 1.49 & 1.56 & 1.25 & 1.25 & 1.56 & 1.57 & 1.56 & 1.50 \\
\hline $6-31 G^{*}$ & 1.37 & 1.49 & 1.55 & 1.37 & 1.47 & 1.55 & 1.23 & 1.23 & 1.56 & 1.55 & 1.55 & 1.48 \\
\hline 6-311G* & 1.37 & 1.49 & 1.55 & 1.37 & 1.48 & 1.55 & 1.23 & 1.23 & 1.56 & 1.55 & 1.55 & 1.48 \\
\hline $6-311 \mathrm{G}^{* *}$ & 1.37 & 1.49 & 1.55 & 1.37 & 1.47 & 1.55 & 1.23 & 1.23 & 1.56 & 1.56 & 1.55 & 1.48 \\
\hline \multicolumn{13}{|l|}{ MP2 } \\
\hline $3-21 \mathrm{G}$ & 1.37 & 1.50 & 1.55 & 1.37 & 1.49 & 1.55 & 1.25 & 1.25 & 1.55 & 1.56 & 1.55 & 1.49 \\
\hline $6-31 \mathrm{G}^{*}$ & 1.36 & 1.47 & 1.53 & 1.36 & 1.46 & 1.53 & 1.23 & 1.23 & 1.53 & 1.53 & 1.53 & 1.46 \\
\hline $6-311 \mathrm{G}^{*}$ & 1.36 & 1.47 & 1.53 & 1.36 & 1.46 & 1.53 & 1.22 & 1.22 & 1.53 & 1.53 & 1.53 & 1.46 \\
\hline $6-311 G^{* *}$ & 1.36 & 1.47 & 1.53 & 1.36 & 1.46 & 1.53 & 1.22 & 1.22 & 1.53 & 1.54 & 1.53 & 1.46 \\
\hline
\end{tabular}

All distances are in $\AA$. (a) Angles of diketopiperazine bonds. (b) Angles of side chain bonds. ${ }^{a}$ Crystal structure obtained from [Cotrait, M.; Leroy, F. Cryst. Struct. Comm. 1979, 8, 819-822] 
Supplement Table 5. Bond Angles of Cyclo(L-Pro-Gly), Diketopiperazine Ring.

\begin{tabular}{|c|c|c|c|c|c|c|c|c|}
\hline & \multicolumn{6}{|c|}{ Diketopiperazine Ring } & \multicolumn{2}{|c|}{ Amide } \\
\hline & $\begin{array}{l}\bar{U} \\
\underbrace{\prime} \\
\delta^{\prime} \\
\underline{1} \\
\bar{Z}\end{array}$ & $\begin{array}{l}Z_{-1}^{N} \\
U^{\prime} \\
\underbrace{\prime} \\
U^{\prime}\end{array}$ & 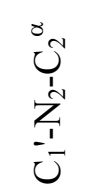 & $\begin{array}{l}\underbrace{N}_{1} \\
\underbrace{\prime} \\
\bigcup_{1} \\
Z^{\prime} \\
Z\end{array}$ & $\begin{array}{l}\vec{Z} \\
\overrightarrow{-1} \\
\underbrace{\prime}_{1} \\
\delta^{\prime} \\
U^{N}\end{array}$ & 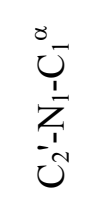 & $\begin{array}{c}0_{1}^{N} \\
-1 \\
\underbrace{}_{1} \\
1 \\
Z\end{array}$ & $\begin{array}{l}\varrho_{0}^{\circ} \\
u^{\prime} \\
U_{1}^{\prime} \\
Z^{\prime}\end{array}$ \\
\hline Cryst & 0.3 & 113.9 & 122.5 & 112.2 & 114.2 & 122.4 & 124.0 & 123.4 \\
\hline CVFI & 6.5 & 119.2 & 121.1 & 117.5 & 118.1 & 121.8 & 118.2 & 122.5 \\
\hline \multicolumn{9}{|l|}{ RHF } \\
\hline $3-21 G$ & 9.1 & 111.0 & 123.2 & 110.0 & 112.4 & 123.3 & 124.0 & 124.6 \\
\hline $6-31$ & 1.0 & 112.4 & 124.0 & 111.1 & 113.7 & 123.5 & 123.6 & 124.6 \\
\hline $6-311$ & 111.1 & 113.1 & 123.8 & 111.3 & 113.5 & 123.3 & 123.7 & 124.7 \\
\hline 6-311 & 111.1 & 112.9 & 123.8 & 111.3 & 113.5 & 123.4 & 123.7 & 124.7 \\
\hline \multicolumn{9}{|l|}{ B3LYP } \\
\hline $3-21 G$ & 0.2 & 112.4 & 122.9 & 110.5 & 111.9 & 123.2 & 124.5 & 124.5 \\
\hline $6-3$ & 1.4 & 113.1 & 124.1 & 111.6 & 113.3 & 123.6 & 123.7 & 124.4 \\
\hline & 111.4 & 112.9 & 124.1 & 111.8 & 113.3 & 123.6 & 123.9 & 124.7 \\
\hline $6-311 \mathrm{G}^{* *}$ & 111.4 & 112.9 & 124.1 & 111.8 & 113.3 & 123.6 & 123.9 & 124.7 \\
\hline \multicolumn{9}{|c|}{ 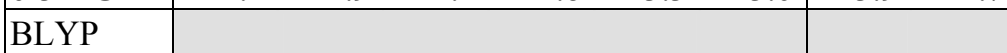 } \\
\hline $3-21 G$ & 0.5 & 112.3 & 122.9 & 110.6 & 111.6 & 123.1 & 124.5 & 124.6 \\
\hline & 1.5 & 113.0 & 124.3 & 111.8 & 113.5 & 123.7 & 123.7 & 124.5 \\
\hline 6-: & 111.6 & 112.8 & 124.3 & 112.1 & 113.3 & 123.7 & 123.9 & 124.8 \\
\hline $6-311 \mathrm{G}^{* *}$ & 111.5 & 112.9 & 124.3 & 112.0 & 113.3 & 123.8 & 123.9 & 124.7 \\
\hline \multicolumn{9}{|l|}{ BVWN } \\
\hline $3-21 G$ & 0.6 & 112.2 & 123.2 & 111.0 & 111.9 & 123.3 & 124.3 & 124.8 \\
\hline 6- & 1.7 & 112.9 & 124.6 & 112.1 & 113.4 & 123.9 & 124.0 & 124.9 \\
\hline $6-311 G^{*}$ & 111.7 & 112.9 & 124.6 & 112.3 & 113.5 & 123.8 & 123.4 & 124.9 \\
\hline 6-311 & 111.7 & 112.9 & 124.6 & 112.3 & 113.5 & 123.8 & 123.9 & 124.8 \\
\hline \multicolumn{9}{|l|}{ MP2 } \\
\hline & 9.6 & 112.2 & 122.2 & 109.2 & 111.5 & 122.5 & 124.6 & 124.7 \\
\hline $6-31 G^{*}$ & 110.4 & 112.3 & 122.9 & 109.9 & 112.4 & 122.6 & 124.1 & 124.6 \\
\hline $6-311 G^{*}$ & 110.7 & 111.9 & 122.5 & 109.8 & 111.9 & 122.4 & 124.4 & 124.9 \\
\hline $6-311 \mathrm{G}^{* *}$ & 110.7 & 111.8 & 122.5 & 109.7 & 111.8 & 122.4 & 124.5 & 124.9 \\
\hline
\end{tabular}

All angles are in degrees. ${ }^{a}$ Crystal structure obtained from [Cotrait, M.; Leroy, F. Cryst. Struct. Comm. 1979, 8, 819-822] 
Supplement Table 6. Bond Dihedral Angles of Cyclo(L-Pro-Gly).

\begin{tabular}{|c|c|c|c|c|c|c|c|c|c|c|c|}
\hline & \multicolumn{6}{|c|}{ Diketopiperazine Ring } & \multicolumn{5}{|c|}{ Proline Side Chain } \\
\hline & $\phi_{1}$ & $\psi_{1}$ & $\omega_{1}$ & $\phi_{2}$ & $\psi_{2}$ & $\omega_{2}$ & $\chi_{11}$ & Q21 & 久3 & $\lambda 41$ & $\chi_{51}$ \\
\hline Crystal $^{\mathrm{a}}$ & 43.9 & & 0.3 & 37.3 & 32.7 & 7.3 & 32.7 & & & & \\
\hline & & 20.6 & & & 19.8 & -1.0 & & & & & 77 \\
\hline $\mathrm{HF}$ & & 20.6 & 2.0 & 18.0 & 19.9 & -1.0 & & & & & \\
\hline & & & 2.5 & & & 3.6 & & & & & 17.0 \\
\hline & & & & & & & & & & & 8.3 \\
\hline & & & 2.6 & & & 3.5 & & & & & 8.3 \\
\hline & 6 & 42.1 & -4.0 & & 43.3 & -5.0 & & & & & 01 \\
\hline & & 36.6 & -0.6 & 6.9 & 36.4 & 04 & & & & & \\
\hline & & & & & & & & & & & 4.6 \\
\hline & & 36.7 & & & & 0.5 & & & & & 7.3 \\
\hline & 5 & 42.3 & -5.4 & 2 & & -7.7 & 9 & & & & 8.0 \\
\hline & 5.4 & 36.9 & -1.8 & -36.0 & 36.4 & -1.2 & 5 & & & & 70 \\
\hline & & 36.7 & & & & & & & & & \\
\hline & & 36 & 1.2 & 2 & 35 & 0.0 & & & & & 8. \\
\hline & 7.5 & 42.0 & -5.2 & 3 & 42.7 & -5.6 & 4 & & & & 7.3 \\
\hline & & 35.7 & -0.4 & -35.7 & 34.0 & 1.4 & 5 & & & & 8.2 \\
\hline & & & & & & 1.8 & & & & & 8.2 \\
\hline & & & 6 & & 34.1 & 1.4 & & & & & \\
\hline & & 43.9 & J. & -4 & 47.2 & -7.3 & & & & & 4.5 \\
\hline & & 40.3 & 0. & & 43.3 & -3.4 & & & & & 9.3 \\
\hline & & & 0.9 & & 44.6 & -3.7 & & & & & 9.3 \\
\hline & & & & & 44.7 & -3.4 & & & 10 & & 9.3 \\
\hline MP & .9 & 38.5 & 0.3 & -37.3 & 32.7 & 7.3 & & & & & \\
\hline $3-2$ & .7 & 20.6 & -2.1 & -18.0 & 19.8 & -1.0 & 22.2 & -37.0 & 36.9 & & 1.3 \\
\hline & & 20.6 & -2.0 & -18.0 & 19.9 & -1.0 & 22.3 & -37.1 & 37.0 & -24.3 & 1.3 \\
\hline 011 & -40.3 & 35.9 & 2.5 & -39.2 & 35.0 & 3.6 & 23.1 & -37.6 & 37.1 & -23.9 & 0.5 \\
\hline
\end{tabular}


Supplement Table 7. Bond Lengths of Cyclo(L-Pro-L-Ala).

\begin{tabular}{|c|c|c|c|c|c|c|c|c|c|c|c|c|c|}
\hline & \multicolumn{6}{|c|}{ Diketopiperazine ring } & \multicolumn{2}{|c|}{ Carbonyl } & \multicolumn{4}{|c|}{ Proline } & \multirow{2}{*}{ 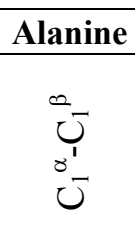 } \\
\hline & $\begin{array}{l}\overrightarrow{z_{1}} \\
\dot{u}\end{array}$ & $\begin{array}{l}U_{1}^{0} \\
\bar{Z}\end{array}$ & $\begin{array}{l}\bar{U} \\
\bar{U} \\
\bar{U}\end{array}$ & $\begin{array}{l}Z_{1}^{N} \\
u\end{array}$ & $\begin{array}{l}\delta^{N} \\
\stackrel{1}{1} \\
Z^{\prime}\end{array}$ & $\begin{array}{l}U_{1}^{N} \\
\tilde{U}^{N}\end{array}$ & $\begin{array}{l}0 \\
1 \\
0 \\
0\end{array}$ & $\begin{array}{l}O_{1}^{N} \\
ن \\
ن\end{array}$ & $\begin{array}{l}\infty \\
U^{\prime} \\
\delta^{\prime} \\
U\end{array}$ & $\begin{array}{l}U_{J}^{\prime} \\
\sigma^{\prime} \\
U\end{array}$ & $\begin{array}{l}\infty \\
U_{1}^{\prime} \\
\sigma^{\prime} \\
U\end{array}$ & $\begin{array}{l}U^{\prime} \\
\tilde{U}^{\prime}\end{array}$ & \\
\hline Crystal $^{\mathrm{a}}$ & 1.32 & 1.47 & 1.51 & 1.33 & 1.47 & 1.53 & 1.23 & 1.23 & 1.53 & 1.52 & 1.53 & 1.52 & 1.53 \\
\hline CVFF & 1.35 & 1.50 & 1.55 & 1.34 & 1.49 & 1.58 & 1.23 & 1.23 & 1.54 & 1.52 & 1.54 & 1.52 & 1.54 \\
\hline \multicolumn{14}{|l|}{ RHF } \\
\hline $3-21 \mathrm{G}$ & 1.34 & 1.47 & 1.51 & 1.35 & 1.47 & 1.53 & 1.22 & 1.26 & 1.54 & 1.55 & 1.54 & 1.55 & 1.54 \\
\hline $6-31 G^{*}$ & 1.34 & 1.46 & 1.52 & 1.35 & 1.46 & 1.53 & 1.20 & 1.24 & 1.53 & 1.53 & 1.53 & 1.53 & 1.53 \\
\hline $6-311 G^{*}$ & 1.34 & 1.46 & 1.52 & 1.35 & 1.46 & 1.53 & 1.19 & 1.23 & 1.53 & 1.53 & 1.53 & 1.53 & 1.53 \\
\hline 6-311G** & 1.34 & 1.46 & 1.52 & 1.35 & 1.46 & 1.53 & 1.19 & 1.23 & 1.53 & 1.53 & 1.53 & 1.53 & 1.53 \\
\hline \multicolumn{14}{|c|}{$\begin{array}{ll} \\
\end{array}$} \\
\hline $3-21 G$ & 1.36 & 1.48 & 1.53 & 1.37 & 1.48 & 1.55 & 1.24 & 1.24 & 1.54 & 1.55 & 1.54 & 1.55 & 1.54 \\
\hline 6-31G* & 1.35 & 1.47 & 1.53 & 1.36 & 1.47 & 1.54 & 1.22 & 1.23 & 1.54 & 1.54 & 1.54 & 1.54 & 1.54 \\
\hline $6-311 \mathrm{G}^{*}$ & 1.35 & 1.47 & 1.53 & 1.36 & 1.47 & 1.54 & 1.22 & 1.22 & 1.53 & 1.54 & 1.53 & 1.54 & 1.53 \\
\hline $6-311 G^{* *}$ & 1.35 & 1.47 & 1.53 & 1.36 & 1.47 & 1.54 & 1.22 & 1.22 & 1.53 & 1.54 & 1.53 & 1.54 & 1.53 \\
\hline \multicolumn{14}{|l|}{ BLYP } \\
\hline $3-21 G$ & 1.37 & 1.50 & 1.55 & 1.38 & 1.50 & 1.57 & 1.25 & 1.26 & 1.57 & 1.57 & 1.57 & 1.57 & 1.57 \\
\hline 6-31G* & 1.37 & 1.49 & 1.54 & 1.38 & 1.48 & 1.56 & 1.24 & 1.24 & 1.55 & 1.55 & 1.55 & 1.55 & 1.55 \\
\hline 6-311 & 1.37 & 1.49 & 1.54 & 1.37 & 1.48 & 1.56 & 1.23 & 1.23 & 1.55 & 1.55 & 1.55 & 1.55 & 1.55 \\
\hline $6-311 \mathrm{G}^{* *}$ & 1.37 & 1.49 & 1.54 & 1.37 & 1.48 & 1.56 & 1.23 & 1.23 & 1.55 & 1.55 & 1.55 & 1.55 & 1.55 \\
\hline \multicolumn{14}{|l|}{ BVWN } \\
\hline $3-21 G$ & 1.37 & 1.50 & 1.55 & 1.38 & 1.50 & 1.57 & 1.25 & 1.26 & 1.55 & 1.57 & 1.55 & 1.57 & 1.55 \\
\hline 6-31G* & 1.37 & 1.48 & 1.54 & 1.38 & 1.48 & 1.56 & 1.24 & 1.24 & 1.55 & 1.55 & 1.55 & 1.55 & 1.55 \\
\hline $6-311 G^{*}$ & 1.37 & 1.48 & 1.54 & 1.37 & 1.48 & 1.56 & 1.23 & 1.23 & 1.54 & 1.55 & 1.54 & 1.55 & 1.54 \\
\hline $6-311 \mathrm{G}^{* *}$ & 1.37 & 1.49 & 1.54 & 1.37 & 1.48 & 1.56 & 1.23 & 1.23 & 1.54 & 1.55 & 1.54 & 1.55 & 1.54 \\
\hline \multicolumn{14}{|l|}{ MP2 } \\
\hline $3-21 G$ & 1.37 & 1.49 & 1.54 & 1.38 & 1.49 & 1.55 & 1.25 & 1.26 & 1.55 & 1.56 & 1.55 & 1.56 & 1.55 \\
\hline 6-31G* & 1.35 & 1.46 & 1.52 & 1.36 & 1.46 & 1.53 & 1.23 & 1.24 & 1.53 & 1.53 & 1.53 & 1.53 & 1.53 \\
\hline $6-311 \mathrm{G}^{*}$ & 1.35 & 1.46 & 1.52 & 1.36 & 1.46 & 1.53 & 1.22 & 1.23 & 1.53 & 1.53 & 1.53 & 1.53 & 1.53 \\
\hline 6-311G** & 1.36 & 1.46 & 1.52 & 1.36 & 1.46 & 1.53 & 1.22 & 1.23 & 1.53 & 1.52 & 1.53 & 1.52 & 1.53 \\
\hline
\end{tabular}

All bond lengths are in $\AA$. ${ }^{\text {a }}$ Crystal structure obtained from [Cotrait and Leroy Cotrait, M.; Leroy, F. Cryst. Struct. Comm. 1979, 8, 819]. 
Supplement Table 8. Bond Angles of Cyclo(L-Pro-L-Ala).

\begin{tabular}{|c|c|c|c|c|c|c|c|c|c|c|c|c|c|c|}
\hline & \multicolumn{6}{|c|}{ Diketopiperazine Ring } & \multicolumn{2}{|c|}{ Amide } & \multicolumn{6}{|c|}{ Side Chains } \\
\hline & $\begin{array}{l}\bar{U} \\
J_{1} \\
U^{\prime} \\
\vdots \\
Z \\
Z\end{array}$ & $\begin{array}{l}Z_{-1}^{N} \\
U^{\prime} \\
\underbrace{\prime} \\
U\end{array}$ & 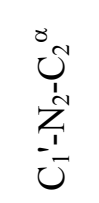 & $\begin{array}{l}\bigcup^{\prime} \\
\mho^{\prime} \\
\bigcup_{1} \\
Z \\
Z\end{array}$ & $\begin{array}{l}Z_{1}^{\prime} \\
\mathcal{U}^{\prime} \\
\mho^{\prime} \\
\sigma^{\prime}\end{array}$ & 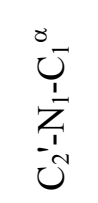 & $\begin{array}{c}0^{N} \\
-1 \\
-1 \\
1-1 \\
Z\end{array}$ & 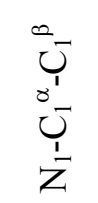 & 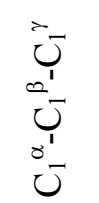 & $\begin{array}{l}U_{1}^{\infty} \\
\vdots \\
U^{\prime} \\
\infty^{\prime} \\
U^{\prime}\end{array}$ & $\begin{array}{l}\vec{Z} \\
\mathbf{D}^{1} \\
U^{\prime} \\
\dot{U} \\
\dot{U}\end{array}$ & $\begin{array}{l}\mho_{-}^{\circ} \\
U_{1} \\
Z_{1} \\
0^{\prime} \\
U_{-}^{\prime}\end{array}$ & $\begin{array}{l}e^{0} \\
u^{\prime} \\
\sigma^{\prime} \\
U^{\prime} \\
Z\end{array}$ & $\begin{array}{l}U_{0}^{\prime} \\
0^{\prime} \\
U_{0}^{\prime} \\
\sigma^{\prime} \\
U^{\prime}\end{array}$ \\
\hline Crystal $^{\mathrm{a}}$ & 111.6 & 114.8 & 123.1 & 109.9 & 114.4 & 122.0 & 123.8 & 123.8 & 102.9 & 102.8 & 104.5 & 103.9 & 112.0 & 110.0 \\
\hline CVFF & 116.8 & 119.8 & 122.0 & 116.8 & 118.3 & 122.0 & 117.9 & 121.4 & 100.4 & 103.1 & 104.5 & 103.0 & 113.7 & 105.6 \\
\hline \multicolumn{15}{|l|}{ RHF } \\
\hline $3-21 G$ & 10.2 & 113.1 & 124.5 & 109.2 & 113.6 & 124.1 & 124.2 & 123.7 & 103.1 & 102.8 & 103.8 & 102.8 & 113.3 & 110.0 \\
\hline $6-31 \mathrm{G}^{*}$ & 112.4 & 114.1 & 124.8 & 109.9 & 114.5 & 124.7 & 123.7 & 123.4 & 103.0 & 102.4 & 104.1 & 103.2 & 112.9 & 110.1 \\
\hline $6-311 G^{*}$ & 112.4 & 113.9 & 124.5 & 109.9 & 114.2 & 124.5 & 123.8 & 123.6 & 103.8 & 102.4 & 104.5 & 103.2 & 112.8 & 110.1 \\
\hline $6-311 \mathrm{G}^{* *}$ & 112.4 & 113.9 & 124.5 & 109.9 & 114.3 & 124.5 & 123.8 & 123.5 & 103.0 & $102 . .4$ & 104.1 & 103.2 & 112.9 & 110.2 \\
\hline \multicolumn{15}{|l|}{ B3LYP } \\
\hline $3-21 G$ & 110.6 & 112.7 & 124.5 & 109.9 & 113.2 & 124.0 & 124.6 & 123.9 & 103.2 & 103.4 & 104.2 & 103.0 & 113.1 & 109.3 \\
\hline 6-31G* & 112.7 & 114.0 & 124.8 & 110.4 & 114.6 & 125.0 & 123.8 & 123.2 & 103.0 & 102.7 & 104.3 & 103.5 & 112.7 & 110.4 \\
\hline $6-311 G^{*}$ & 112.6 & 113.7 & 124.5 & 110.5 & 114.3 & 124.8 & 124.0 & 123.5 & 103.1 & 102.8 & 104.3 & 103.4 & 112.6 & 110.3 \\
\hline 6-311G** & 112.5 & 113.7 & 124.5 & 110.5 & 114.3 & 124.8 & 124.0 & 123.5 & 103.1 & 102.8 & 104.3 & 103.4 & 112.7 & 110.4 \\
\hline \multicolumn{15}{|l|}{ BLYP } \\
\hline $3-21 G$ & 110.6 & 112.3 & 124.6 & 110.1 & 112.9 & 123.9 & 124.8 & 124.0 & 103.4 & 103.7 & 104.4 & 103.2 & 112.8 & 110.5 \\
\hline 6-31G* & 112.8 & 114.0 & 125.9 & 110.5 & 114.6 & 125.1 & 123.8 & 123.2 & 103.1 & 103.0 & 104.6 & 103.7 & 112.5 & 110.3 \\
\hline $6-311 \mathrm{G}^{*}$ & 112.8 & 113.6 & 125.7 & 110.7 & 114.3 & 125.0 & 124.0 & 123.5 & 103.1 & 103.1 & 104.7 & 103.7 & 112.3 & 110.3 \\
\hline $6-311 \mathrm{G}^{* *}$ & 112.7 & 113.6 & 125.7 & 110.7 & 114.3 & 124.9 & 124.0 & 123.5 & 103.2 & 103.1 & 104.6 & 103.7 & 112.4 & 110.4 \\
\hline \multicolumn{15}{|l|}{ BVWN } \\
\hline $3-21 G$ & 111.1 & 112.5 & 124.9 & 110.2 & 113.1 & 124.3 & 124.6 & 123.9 & 103.4 & 103.7 & 104.3 & 103.0 & 113.0 & 110.6 \\
\hline 6-31G* & 112.9 & 114.0 & 126.0 & 110.6 & 114.6 & 125.2 & 123.7 & 123.3 & 103.0 & 102.9 & 104.4 & 103.5 & 112.6 & 110.4 \\
\hline $6-311 \mathrm{G}^{*}$ & 112.9 & 113.7 & 125.8 & 110.7 & 114.4 & 125.1 & 123.8 & 123.6 & 103.1 & 102.9 & 104.5 & 103.5 & 112.5 & 110.3 \\
\hline $6-311 \mathrm{G}^{* *}$ & 112.8 & 113.7 & 125.9 & 110.7 & 114.4 & 125.0 & 123.8 & 123.6 & 103.1 & 102.9 & 104.5 & 103.5 & 112.5 & 110.3 \\
\hline \multicolumn{15}{|l|}{ MP2 } \\
\hline $3-21 G$ & 109.7 & 112.2 & 123.7 & 108.7 & 112.6 & 123.2 & 124.9 & 124.2 & 103.1 & 103.5 & 103.9 & 102.4 & 113.3 & 110.0 \\
\hline 6-31G* & 111.9 & 113.4 & 124.2 & 109.0 & 113.6 & 124.3 & 123.2 & 123.5 & 102.6 & 102.6 & 103.8 & 102.6 & 113.1 & 110.1 \\
\hline $6-311 G^{*}$ & 112.2 & 113.0 & 123.8 & 109.1 & 113.0 & 124.2 & 124.5 & 123.8 & 102.7 & 102.4 & 103.6 & 102.5 & 113.0 & 110.1 \\
\hline $6-311 \mathrm{G}^{* *}$ & 112.3 & 113.1 & 123.8 & 109.1 & 113.0 & 124.3 & 124.6 & 123.8 & 102.5 & 102.3 & 103.5 & 102.5 & 113.1 & 110.0 \\
\hline
\end{tabular}

All angles are in degrees. ${ }^{\text {a }}$ Crystal structure obtained from reference from [Cotrait and Leroy Cotrait, M.; Leroy, F. Cryst. Struct. Comm. 1979, 8, 819]. 
Supplement Table 9. Bond Dihedral Angles of Cyclo(L-Pro-L-Ala).

\begin{tabular}{|c|c|c|c|c|c|c|c|c|c|c|c|c|}
\hline & $\phi_{1}$ & $\psi_{1}$ & $\omega_{1}$ & $\phi_{2}$ & $\psi_{2}$ & $\omega_{2}$ & $\chi_{11}$ & $\chi_{21}$ & $\chi_{31}$ & $\chi_{41}$ & $\chi_{51}$ & $\chi_{12}$ \\
\hline Crystal $^{\mathrm{a}}$ & \begin{tabular}{|l|}
-36.8 \\
\end{tabular} & 30.2 & 8.6 & -42.6 & 35.4 & 2.4 & -33.2 & 37.3 & -26.5 & 4.5 & 17.6 & -166.7 \\
\hline CVFF & -15.7 & 18.3 & -2.8 & -14.7 & 17.0 & -1.6 & -36.6 & 38.9 & -24.4 & 0.8 & 22.8 & -140.9 \\
\hline \multicolumn{13}{|l|}{ RHF } \\
\hline $3-21 G$ & \begin{tabular}{|l|}
-39.4 \\
\end{tabular} & 37.6 & 0.8 & -39.9 & 38.0 & 0.0 & -32.6 & 37.7 & -27.8 & 7.5 & 16.0 & -161.0 \\
\hline 6-31G* & $\mid-33.0$ & 28.1 & 8.3 & -40.6 & 34.7 & 0.0 & -34.1 & 37.4 & -25.8 & 4.2 & 19.1 & -163.6 \\
\hline $6-311 G^{*}$ & -34.0 & 28.7 & 8.6 & -41.3 & 35.3 & 0.3 & -34.1 & 37.6 & -26.3 & 4.7 & 18.8 & -164.8 \\
\hline 6-311G* & -33.9 & 28.8 & 8.6 & -41.6 & 35.3 & 0.0 & -34.1 & 37.7 & -26.5 & 4.9 & 18.6 & -164.6 \\
\hline \multicolumn{13}{|l|}{ B3LYP } \\
\hline $3-21 G$ & 8.1 & 39.5 & 8.3 & -37.2 & 38.6 & -2.4 & -30.7 & 36.6 & -27.5 & 8.5 & 14.2 & -157.8 \\
\hline $6-31 G^{*}$ & -31.2 & 28.7 & -3.0 & -36.8 & 33.5 & -1.3 & -33.6 & 36.7 & -25.3 & 4.0 & 18.9 & -159.6 \\
\hline $6-311 G^{*}$ & -33.4 & 29.8 & 5.1 & -37.7 & 33.6 & 0.0 & -33.5 & 36.8 & -25.4 & 4.1 & 18.8 & -160.9 \\
\hline 6-311 & -33.2 & 30.2 & 4.6 & -37.5 & 33.6 & 0.0 & -33.5 & 36.9 & -25.6 & 4.4 & 18.5 & -160.7 \\
\hline \multicolumn{13}{|l|}{ BLYP } \\
\hline $3-21 G$ & 8.1 & 40.9 & -4.9 & -36.1 & 39.0 & -3.3 & -29.8 & 36.2 & -27.9 & 9.5 & 12.9 & -156.8 \\
\hline $6-31 G^{*}$ & -30.5 & 28.4 & 4.5 & -36.0 & 33.0 & -1.6 & -33.5 & 36.5 & -24.9 & 3.6 & 19.1 & -159.0 \\
\hline 6-311 & -32.5 & 29.2 & 4.8 & -36.8 & 32.9 & 0.0 & -33.4 & 36.3 & -24.7 & 3.5 & 19.1 & -160.2 \\
\hline 6-311G** & -32.7 & 29.7 & 4.3 & -36.5 & 32.9 & 0.0 & -33.4 & 36.4 & -25.0 & 3.8 & 18.8 & -159.8 \\
\hline \multicolumn{13}{|l|}{ BVWN } \\
\hline $3-21 G$ & 36.6 & 38.8 & -3.5 & -36.0 & 38.2 & -3.4 & -30.2 & 35.8 & -26.8 & 8.1 & 14.1 & -160.9 \\
\hline $6-31 G^{*}$ & 30.6 & 27.6 & 5.5 & -36.4 & 32.5 & -0.9 & -33.2 & 35.7 & -24.0 & 2.9 & 19.3 & -165.6 \\
\hline $6-311 G^{*}$ & -32.4 & 28.3 & 5.6 & -36.8 & 32.2 & 0.3 & -33.2 & 35.5 & -23.7 & 2.6 & 19.5 & -167.5 \\
\hline $6-311 \mathrm{G}^{* *}$ & -32.2 & 28.8 & 5.1 & -36.5 & 32.2 & 0.3 & -33.1 & 35.7 & -24.0 & 3.0 & 19.2 & -167.8 \\
\hline \multicolumn{13}{|l|}{ MP2 } \\
\hline $3-21 G$ & 9.9 & 42.2 & -2.7 & -41.1 & 43.1 & -4.3 & -33.2 & 37.3 & -26.5 & 4.5 & 17.6 & -166.7 \\
\hline 6-31G* & -34.0 & 30.9 & 7.5 & -43.8 & 39.5 & -3.0 & -36.6 & 38.9 & -24.4 & 0.8 & 22.8 & -140.9 \\
\hline 6-311G* & -34.8 & 31.1 & 8.5 & -45.5 & 40.5 & -2.8 & -32.6 & 37.7 & -27.8 & 7.5 & 16.0 & -161.0 \\
\hline $6-311 G^{* *}$ & -34.5 & 29.9 & 10.0 & -46.2 & 40.2 & -2.3 & -34.1 & 37.4 & -25.8 & 4.2 & 19.1 & -163.6 \\
\hline
\end{tabular}

All angles are in degrees. ${ }^{\mathrm{a}}$ Crystal structure obtained from reference from [Cotrait and Leroy Cotrait, M.; Leroy, F. Cryst. Struct. Comm. 1979, 8, 819]. 
Supplement Table 10. Bond Lengths of Cyclo(L-Pro-L-Leu).

\begin{tabular}{|c|c|c|c|c|c|c|c|c|c|c|c|c|c|c|c|c|}
\hline & \multicolumn{6}{|c|}{ Diketopiperazine Ring } & \multicolumn{2}{|c|}{ Carbonyl } & \multicolumn{4}{|c|}{ Proline Side Chain } & \multicolumn{4}{|c|}{ Leucine Side Chain } \\
\hline & $\begin{array}{l}\bar{Z} \\
\dot{N} \\
\bar{U}\end{array}$ & 薄 & $\begin{array}{l}U_{1}^{-} \\
\mho^{\prime} \\
U^{\prime}\end{array}$ & U & $\begin{array}{l}U_{1}^{8} \\
\dot{1} \\
Z^{\prime}\end{array}$ & 'ָ' & $\begin{array}{l}0 \\
-1 \\
-1 \\
U\end{array}$ & $\begin{array}{l}0_{-1}^{N} \\
\text { Un }\end{array}$ & $\begin{array}{l}e^{\infty} \\
U^{\prime} \\
\sigma^{\prime} \\
U\end{array}$ & $\begin{array}{l}U^{\prime} \\
\tilde{U}^{\prime} \\
\dot{U}\end{array}$ & $\begin{array}{l}\underbrace{}_{-1} \\
U^{\prime} \\
ن\end{array}$ & $\begin{array}{l}\overrightarrow{Z_{1}} \\
\bar{U}^{\prime}\end{array}$ & $\begin{array}{l}\underbrace{\infty} \underbrace{\prime} \\
\mho^{\prime} \\
U^{\prime}\end{array}$ & $\begin{array}{l}U_{0}^{\prime} \\
\infty^{\prime} \\
\sigma^{\prime}\end{array}$ & $\begin{array}{l}\vec{\omega} \\
\bigcup^{\prime} \\
\check{U}^{\prime}\end{array}$ & 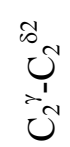 \\
\hline & & 46 & & 34 & 1.46 & 1.52 & 23 & 1.24 & 52 & 1.49 & 1.51 & 1.48 & 1.54 & 1.53 & 1.5 & 1.53 \\
\hline CVI & & & & & 49 & & 23 & & & 154 & 1.53 & & 1.56 & 1.56 & 1.5 & .54 \\
\hline \multicolumn{17}{|l|}{ RHF } \\
\hline 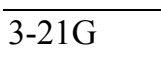 & 5 & 47 & 52 & 35 & 1.47 & 1.53 & 22 & 1.22 & 1.54 & 1.55 & 1.54 & 1.47 & 1.54 & 1.54 & 1.5 & 1.54 \\
\hline & & 1.46 & .52 & 1.35 & 1.46 & 1.53 & 20 & 1.20 & 1.54 & 1.53 & 1.53 & 1.46 & 1.53 & 1.54 & 1.5 & 1.53 \\
\hline & 35 & 1.46 & 1.53 & 1.36 & 1.47 & 1.55 & 19 & 1.20 & 1.54 & 1.53 & 1.53 & 1.46 & 1.53 & 1.54 & 1.5 & 1.53 \\
\hline & & 1.46 & 1.53 & 1.36 & 1.47 & 1.55 & 19 & 1.20 & 1.54 & 1.53 & 1.53 & 1.46 & 1.53 & 1.54 & 1.5 & .53 \\
\hline \multicolumn{17}{|l|}{ B3LYP } \\
\hline $221 C$ & 6 & 48 & 54 & 1.36 & 1.49 & 1.55 & 24 & 1.24 & 55 & 1.56 & 1.56 & 1.48 & 1.54 & 1.55 & 1.5 & 1.55 \\
\hline & & 1.47 & 54 & 1.36 & 1.47 & & 22 & 1.23 & 54 & 1.54 & 1.54 & 1.47 & 1.54 & 1.54 & 1.5 & 1.54 \\
\hline & & 1.47 & & 1.36 & 1.47 & & 22 & 1.22 & 1.54 & 1.54 & 1.54 & 1.47 & 1.53 & 1.54 & 1.5 & 1.53 \\
\hline 6-311 & 6 & 1.47 & 1.53 & 1.36 & 1.47 & 1.55 & 22 & 1.22 & 1.54 & 1.54 & 1.54 & 1.47 & 1.53 & 1.54 & 1.5 & 1.54 \\
\hline \multicolumn{17}{|l|}{ BLYP } \\
\hline & & 50 & 56 & 38 & 1.51 & 1.57 & 25 & 1.26 & 56 & 1.57 & 1.56 & 1.50 & 1.56 & 1.56 & 1.5 & 1.56 \\
\hline & & 1.49 & 1.55 & 1.37 & 1.49 & & 24 & 1.24 & 1.56 & 1.56 & 1.55 & 1.48 & 1.55 & 1.56 & 1.5 & 1.56 \\
\hline & 37 & 1.49 & 1.55 & 1.37 & 1.49 & 1.57 & 23 & 1.23 & 1.56 & 1.55 & 1.55 & 1.48 & 1.56 & 1.56 & 1.5 & 1.56 \\
\hline $6-311 G^{*}$ & & 1.49 & 1.55 & 1.37 & 1.49 & 1.57 & 23 & 1.23 & 1.56 & 1.55 & 1.55 & 1.48 & 1.56 & 1.56 & 1.5 & 1.55 \\
\hline \multicolumn{17}{|l|}{ BVWN } \\
\hline & & 50 & 1.50 & 1.38 & 1.50 & 1.57 & 25 & 1.26 & 56 & 1.57 & 1.56 & 1.50 & 1.55 & 1.56 & 1.5 & .56 \\
\hline & & 1.49 & 1.55 & 1.37 & 1.48 & & 24 & 1.24 & 1.56 & 1.55 & 1.55 & 1.48 & 1.55 & 1.55 & 1.5 & .55 \\
\hline & & 1.49 & 1.55 & 1.37 & 1.48 & 1.57 & 1.23 & 1.23 & 1.55 & 1.55 & 1.54 & 1.48 & 1.54 & 1.55 & 1.5 & 1.55 \\
\hline 6-311 & & 1.49 & 1.55 & 1.37 & 1.48 & 1.57 & 1.23 & 1.23 & 1.55 & 1.55 & 1.55 & 1.48 & 1.55 & 1.55 & 1.5 & 1.55 \\
\hline \multicolumn{17}{|l|}{ MP2 } \\
\hline & & 50 & .04 & 1.5 & 1.49 & $1 .$. & 1.26 & 1.26 & 55 & 1.5 & 1.55 & 1.49 & 1.54 & . 55 & 1.5 & .55 \\
\hline & & 1.47 & 1.53 & 1.36 & 1.46 & 1. & 1.23 & 1.24 & 1.53 & 1.53 & 1.53 & 1.46 & 1.53 & 1.53 & 1.5 & 1.53 \\
\hline $6-311 \mathrm{G}^{*}$ & 1.36 & 1.47 & 1.53 & 1.36 & 46 & 1 & 1.22 & 1.23 & 1.53 & 1.53 & 1.53 & 1.46 & 1.53 & 1.53 & 1.5 & 1.53 \\
\hline
\end{tabular}

All distances are in $\AA .{ }^{a}$ Crystal structure obtained from [Karle, I. L. J. Am. Chem. Soc. 1972, 94, 81.] 
Supplement Table 11a. Bond Angles of Cyclo(L-Pro-L-Leu), Diketopiperazine Ring.

\begin{tabular}{|c|c|c|c|c|c|c|c|c|}
\hline \multirow[b]{2}{*}{ (a) } & \multicolumn{6}{|c|}{ Diketopiperazine Ring } & \multicolumn{2}{|c|}{ Amide } \\
\hline & $\begin{array}{l}\bar{U} \\
\bar{U} \\
\sigma^{\prime} \\
U_{1} \\
U^{\prime} \\
\bar{Z}\end{array}$ & $\begin{array}{l}Z_{-1}^{\prime} \\
U^{\prime} \\
\delta^{\prime} \\
J^{\prime}\end{array}$ & $\begin{array}{l}U_{1}^{O} \\
U_{1}^{\prime} \\
Z_{-1}^{\prime} \\
U^{\prime}\end{array}$ & $\begin{array}{l}\underbrace{N}_{1} \\
\underbrace{\prime} \\
\underbrace{}_{1} \\
U_{N} \\
Z\end{array}$ & $\begin{array}{l}\overrightarrow{Z_{1}^{\prime}} \\
\overrightarrow{U^{\prime}} \\
\delta^{\prime} \\
\tilde{U}^{\prime}\end{array}$ & $\begin{array}{l}8 \\
U_{1} \\
\vdots \\
Z \\
-1 \\
-1 \\
ن\end{array}$ & $\begin{array}{c}O_{1} \\
-1 \\
U_{1}^{\prime} \\
Z \\
Z\end{array}$ & $\begin{array}{l}U_{1} \\
\mho^{\prime} \\
U_{1}^{\prime} \\
\bar{Z}\end{array}$ \\
\hline Crysta & 10.9 & 114.0 & 123.0 & 110.7 & 113.9 & 123.4 & 123.2 & 122.1 \\
\hline CVFF & 6.2 & 119.2 & 22.2 & 115.5 & 118.4 & 121.7 & 18.2 & 120.8 \\
\hline \multicolumn{9}{|l|}{ RHF } \\
\hline $3-210$ & 3.5 & 2.2 & 123.8 & 97.7 & 112.7 & 123.3 & 24.2 & 123.9 \\
\hline & 0.2 & 112.6 & 124.3 & 08.3 & 113.3 & 123.5 & 23.8 & 123.6 \\
\hline & 0.3 & 112.4 & 124.0 & 08.4 & 113.0 & 123.4 & 23.9 & 123.8 \\
\hline $6-311 G^{*}$ & .3 & 112.5 & 124.0 & 08.3 & 113.0 & 23.4 & 23.9 & 123.8 \\
\hline \multicolumn{9}{|l|}{ B3LYP } \\
\hline & & 112.2 & 125.0 & 10.5 & 112.5 & 23.2 & 24.6 & 124.1 \\
\hline & 0.6 & 112.6 & 124.7 & 08.9 & 113.4 & 123.6 & 23.9 & 123.6 \\
\hline & 0.6 & 112.4 & 124.6 & 109.1 & 113.0 & 123.6 & 124.1 & 123.9 \\
\hline $6-3110$ & & & 124.6 & & 113.1 & 3.6 & 24.1 & 123.8 \\
\hline \multicolumn{9}{|l|}{ BLYP } \\
\hline $3-21 \mathrm{G}$ & 9.9 & 2.0 & 124.0 & 08.7 & 12.3 & 123.3 & 24.5 & 124.2 \\
\hline & 0.7 & 112.5 & 125.1 & 109.0 & 113.4 & 123.8 & 123.8 & 123.6 \\
\hline & 0.7 & 112.3 & 125.0 & 109.2 & 113.1 & 123.9 & 124.0 & 123.9 \\
\hline $6-3110$ & & 112.4 & 125.0 & 9.2 & 3.1 & 23.9 & 124.0 & 123.8 \\
\hline \multicolumn{9}{|l|}{ BVWN } \\
\hline $3-21 G$ & 9.9 & 112.1 & 123.9 & 108.7 & 112.4 & 123.2 & 24.7 & 124.1 \\
\hline & 0.7 & 112.5 & 125.0 & 109.0 & 113.4 & 123.7 & 123.9 & 123.6 \\
\hline & .7 & 112.3 & 124.8 & 109.2 & 113.0 & 123.7 & 124.1 & 123.9 \\
\hline 6-311 & & 112.4 & & .2 & 3.1 & 3.8 & 24.1 & 123.9 \\
\hline \multicolumn{9}{|l|}{ MP2 } \\
\hline $3-210$ & 9.0 & 111.9 & 123.1 & 107.6 & 112.1 & 122.5 & 124.8 & 124.3 \\
\hline & & 111.9 & 123.6 & 107.7 & 112.4 & 122.7 & 124.3 & 123.9 \\
\hline $6-311 \mathrm{G}^{*}$ & 109.9 & 111.4 & 123.2 & 107.7 & 111.8 & 122.5 & 124.6 & 124.3 \\
\hline
\end{tabular}


Supplement Table 11b. Bond Angles of Cyclo(L-Pro-L-Leu), Side Chains.

\begin{tabular}{|c|c|c|c|c|c|c|c|c|c|}
\hline \multirow[b]{2}{*}{ (b) } & \multicolumn{5}{|c|}{ Proline Side Chain } & \multicolumn{4}{|c|}{ Leucine Side Chain } \\
\hline & $\begin{array}{l}\tilde{U}^{0} \\
\delta^{\prime} \\
U_{1}^{\prime} \\
1 \\
Z\end{array}$ & $\begin{array}{l}U_{0}^{\prime} \\
\infty^{\prime} \\
\underbrace{\prime} \\
\mho^{\prime} \\
J^{\prime}\end{array}$ & $\begin{array}{l}\infty \\
U_{1}^{\prime} \\
u^{\prime} \\
\infty^{\prime} \\
\infty^{\prime}\end{array}$ & $\begin{array}{l}Z_{1}^{\prime} \\
0^{\prime} \\
U_{1}^{\prime} \\
\check{U}\end{array}$ & $\begin{array}{l}\mho \\
U \\
Z \\
Z^{\prime} \\
\infty^{\prime} \\
U\end{array}$ & 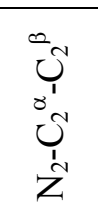 & $\begin{array}{l}\bigcup_{0}^{i} \\
\bigcup^{\prime} \\
\bigcup_{0}^{\prime} \\
\sigma^{\prime}\end{array}$ & $\begin{array}{l}\vec{\infty} \\
\bigcup_{i}^{\prime} \\
\tilde{U}^{\prime} \\
\infty^{\prime} \\
\tilde{U}^{\prime}\end{array}$ & 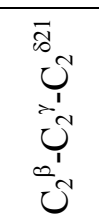 \\
\hline Crystal $^{\mathrm{a}}$ & 103.2 & 103.0 & 107.2 & 101.6 & 112.5 & 110.0 & 114.6 & 107.3 & 107.8 \\
\hline CVFF & 2.1 & 107.1 & 106.1 & 100.8 & 113.8 & 107.7 & 117.9 & 114.1 & 109.8 \\
\hline \multicolumn{10}{|l|}{ RHF } \\
\hline $3-21 G$ & 4.5 & 104.1 & 103.3 & 101.7 & 112.3 & 111.5 & 114.8 & 112.9 & 109.2 \\
\hline 6-31G* & 4.4 & 104.5 & 103.5 & 102.3 & 112.4 & 111.6 & 115.7 & 114.2 & 109.4 \\
\hline 6-311 & 104.4 & 104.4 & 103.5 & 102.3 & 112.4 & 111.7 & 115.8 & 114.2 & 109.5 \\
\hline $6-311 \mathrm{G}^{* *}$ & 104.4 & 104.4 & 103.4 & 102.3 & 112.3 & 111.7 & 115.8 & 114.2 & 109.5 \\
\hline \multicolumn{10}{|l|}{ B3LYP } \\
\hline $3-21 G$ & 4.6 & 104.3 & 103.4 & 102.0 & 111.9 & 111.6 & 115.0 & 112.5 & 109.5 \\
\hline 6-31 & 104.5 & 104.6 & 103.6 & 102.5 & 112.0 & 111.8 & 115.9 & 113.8 & 109.7 \\
\hline 6-311 & 104.5 & 104.7 & 103.7 & 102.5 & 112.0 & 111.8 & 116.0 & 113.8 & 109.8 \\
\hline $6-311 G^{* *}$ & 104.5 & 104.7 & 103.6 & 102.5 & 112.0 & 111.9 & 116.0 & 113.8 & 109.8 \\
\hline \multicolumn{10}{|l|}{ BLYP } \\
\hline $3-21 G$ & 104.6 & 104.4 & 103.5 & 102.2 & 111.7 & 111.7 & 115.2 & 112.6 & 109.6 \\
\hline $6-31 \mathrm{G}^{*}$ & 104.5 & 104.7 & 103.7 & 102.7 & 111.9 & 111.8 & 116.1 & 113.9 & 109.8 \\
\hline $6-311 G^{*}$ & 104.5 & 104.8 & 103.8 & 102.7 & 111.8 & 111.9 & 116.2 & 113.9 & 109.8 \\
\hline $6-311 \mathrm{G}^{* *}$ & 104.5 & 104.8 & 103.8 & 102.6 & 111.8 & 111.9 & 116.2 & 113.8 & 109.8 \\
\hline \multicolumn{10}{|l|}{ BVWN } \\
\hline $3-21 G$ & 104.6 & 104.7 & 103.8 & 102.4 & 111.7 & 111.9 & 115.5 & 113.1 & 109.5 \\
\hline 6-31G* & 104.6 & 105.0 & 104.0 & 102.8 & 111.9 & 111.9 & 111.4 & 114.3 & 109.8 \\
\hline 6-311G* & 104.6 & 105.1 & 104.1 & 102.8 & 111.8 & 111.9 & 116.5 & 114.2 & 109.8 \\
\hline 6-311G** & 104.6 & 105.1 & 104.0 & 102.8 & 111.8 & 111.9 & 116.5 & 114.2 & 109.8 \\
\hline \multicolumn{10}{|l|}{ MP2 } \\
\hline $3-21 G$ & 104.1 & 103.9 & 103.2 & 101.8 & 111.9 & 111.2 & 114.5 & 112.2 & 109.6 \\
\hline $6-31 G^{*}$ & 103.9 & 103.8 & 103.0 & 102.0 & 112.2 & 111.5 & 115.1 & 113.3 & 109.6 \\
\hline $6-311 G^{*}$ & 103.9 & 103.6 & 102.8 & 102.1 & 112.1 & 111.5 & 115.0 & 113.1 & 109.7 \\
\hline
\end{tabular}

All angles are in degrees. (a) Angles of diketopiperazine bonds. (b) Angles of side chain bonds. ${ }^{a}$ Crystal structure obtained from [Karle, I. L. J. Am. Chem. Soc. 1972, 94, 81.] 
Supplement Table 12. Bond Dihedral Angles of Cyclo(L-Pro-L-Leu).

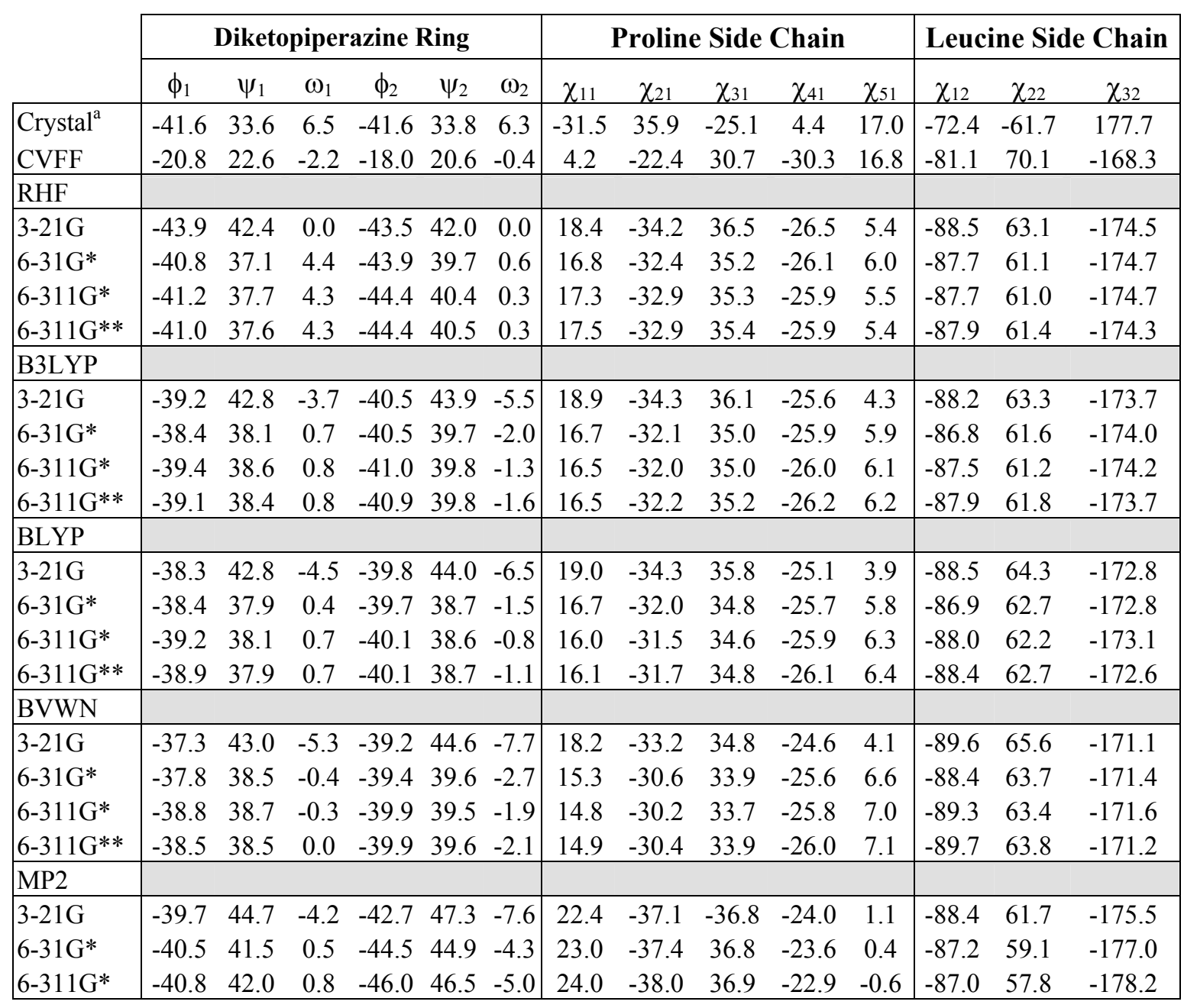

All angles are in degrees. (a) Dihedral angles of diketopiperazine bonds. (b) Dihedral angles of side

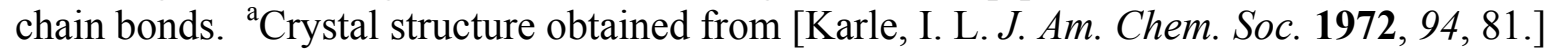

